\title{
Coherent Diffusive Photon Gun for Generating Nonclassical States
}

\author{
M. Thornton®, ${ }^{1, *}$ A. Sakovich, ${ }^{2}$ A. Mikhalychev $\odot,{ }^{2}$ J. D. Ferrer, ${ }^{1}$ P. de la Hoz® ${ }^{1}$ N. Korolkova $\odot,{ }^{1}$ \\ and D. Mogilevtsev ${ }^{2}{ }^{2}$ \\ ${ }^{1}$ School of Physics and Astronomy, University of St Andrews, North Haugh, St Andrews KY16 9SS, \\ United Kingdom \\ ${ }^{2}$ Institute of Physics, Belarus National Academy of Sciences, Nezavisimosti Ave. 68-2, Minsk 220072, Belarus
}

(Received 3 July 2019; revised manuscript received 11 November 2019; published 23 December 2019)

\begin{abstract}
We suggest and discuss a concept of a deterministic integrated source of nonclassical light based on the coherent diffusive photonics, a coherent light flow in a system of dissipatively coupled waveguides. We show how this practical quantum device can be realized with a system of single-mode waveguides laser inscribed in nonlinear glass. We describe a hierarchy of models, from the complete multimode model of the waveguide network to the single mode coupled to a bath, analyze the conditions for validity of the simplest single-mode model and demonstrate feasibility of the generation of bright sub-Poissonian light states merely from a coherent input. Notably, the generation of nonclassical states occurs at the initial stages of the dynamics, and can be accounted for in the linear model that allows us to circumvent the prohibiting computational complexity of the exact full quantum representation.
\end{abstract}

DOI: 10.1103/PhysRevApplied.12.064051

\section{INTRODUCTION}

Engineered loss has already turned into a powerful and intensively researched tool for quantum-state manipulation. With the help of engineered reservoirs, it is possible to drive a target system into a desired state, to generate and protect entanglement, to implement computation, and to transfer quantum states [1-4]. Engineered reservoirs have become a popular and fruitful research direction for QED [5], cold atoms and trapped ions [6,7], Rydberg atoms $[8,9]$, and for superconducting circuits [10-12] due to the large spectra of possibilities to design systems with necessary coupling between components and strong effective nonlinearities. For example, by devising strong self-Kerr and cross-Kerr interactions, and combining them with twophoton conversion and classical driving, it is possible to produce two-photon loss, and in this way create the Schrödinger-cat states and safeguard them against linear loss [10].

It is considerably harder to engineer losses in photonic circuits. Optical nonlinearities are usually quite small and accompanied by rather strong conventional linear loss. However, even in this case there are possibilities to exploit effects of nonlocal loss and devise photonic circuits with quite unusual and potentially very useful features, even in passive optical structures. These possibilities are opened up by a new level of precision and controllability reached in systems of coupled waveguides. For

\footnotetext{
*mt45@st-andrews.ac.uk
}

example, localization of the light in the small part of a perfect Lieb lattice and diffractionless propagation was experimentally demonstrated in a system of single-mode waveguides written in a bulk glass $[13,14]$.

These new experimental advances gave rise to a recently introduced concept of "coherent diffusive photonics" (CDP): light processing in a specifically designed system of single-mode waveguides coupled by common loss reservoirs, as realized in practice in Ref. [15]. Even with linear glass, dissipative coupling enables, for example, light equalization in a waveguide chain: any input state tends to a completely symmetrized state over all the modes. In contrast to the common unitary coupling schemes, CDP schemes are quite robust with respect to variations in the coupling length and strength. Further, CDP allows one to realize an optical router directing light in different arms of the structure by selective excitation of the central control nodes, and CDP can even allow light to be localized in a perfect lattice of dissipatively coupled modes [15].

In this paper we extend the developed CDP schemes to the nonlinear regime, and suggest an alternative integrated CDP-based device exhibiting features of a "photon gun": a deterministic generator of nonclassical states, in particular, sub-Poissonian states (Fig. 1).

It is worth noticing that a true photon gun is still a challenge (for reviews see [16-18]). Due to high technical overhead, the available single-photon sources are not readily implementable, mostly do not have well-defined output mode, and many of them cannot simultaneously satisfy the 

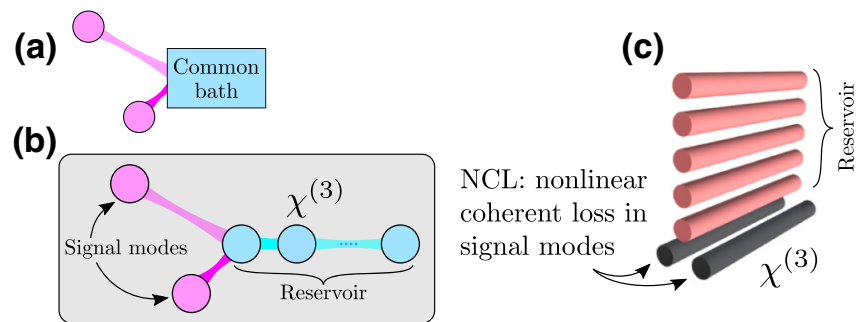

FIG. 1. Deterministic photon gun (PhoG) based on the CDP network of single-mode waveguides written in a bulk of nonlinear glass. (a) Basic scheme behind the PhoG device: two signal modes asymetrically coupled to a common bath. Both modes undergo nonlinear coherent loss (NCL). (b) Implementation of the basic scheme using a network of nonlinear waveguides. The common bath, the reservoir, is implemented as a linear array of further waveguides ("tail"). Signal modes [gray in threedimensional (3D) picture (c)] are dissipatively coupled via this common reservoir. The "tail" waveguides [pink in 3D picture (c)] interact via conventional evanescent coupling.

crucial figures of merit: purity, indistinguishability (often traded for brightness), and efficiency. Only very recently top-end quantum-dot-based sources could demonstrate simultaneously single-photon purity of $\geq 99 \%$, photon indistinguishability of $95 \%-99 \%$ and extraction efficiency of up to $65 \%$ [19]. Other alternative, heralded photon sources based on entangled photon-pair production are probabilistic and deliver high indistinguishability at the cost of low brightness. Therefore, in many quantumtechnology applications, experimentalists revert to attenuated coherent states instead of single photons. This is true for most if not all quantum-cryptography implementations and many others, including quantum simulations and implementations of quantum gates. Dim coherent states are "cheap," easy to handle, and deterministic, however, their Poissonian photon-number statistics represents a serious limitation, resulting in unwanted contribution from multiphoton components. Much better performance can be achieved using light with sub-Poissonian photon statistics as a quasi-single-photon source. For example, the key rates for decoy-state quantum-key distribution (QKD) have been explicitly calculated for different sources, and it was demonstrated that such a quasi-single-photon source can drastically raise the key rate in the decoy-state QKD $[20,21]$.

We demonstrate that the photon-gun device suggested in this paper is potentially able to act either as a deterministic or, upon a slight variation of the scheme, probabilistic quasi-single-photon source. We analyze the possibility of realization of these devices with current technology. Whereas approaching deterministic generation of quasi-single-photon or few-photon strongly subPoissoinian states still seems challenging, we show that bright sub-Poissonian photon gun is completely feasible and can be quite robust with respect to experimental imperfections and noise.

Bright sub-Poissonian PhoG suggested in this paper carries the important advantage of producing nonclassical states from merely coherent input, on demand, and in well-defined temporal and spatial modes. Further, PhoG is a versatile quantum source and can be modified to produce correlated photons and other entangled states at the output. Knowingly, nonclassical photon-number correlations and sub-Poissonian photon statistics can be exploited for quantum sensing, imaging, and other metrology tasks [22,23].

The basic idea underlying our scheme is to create a photonic circuit exhibiting NCL, which is a specific kind of engineered single-photon loss $[24,25]$. The principal circuit, Fig. 1(a), comprises two signal modes asymmetrically coupled to a common loss reservoir and undergoing NCL. The required light-propagation regime can be engineered in different ways. We suggest a nonlinear waveguide network with a chain waveguide structure working as a common reservoir, Fig. 1(b), which allows the NCL to be realized in the superposition of two waveguide modes. We analyze its feasibility under realistic Kerr nonlinearity of nonlinear glass and for realistic waveguide parameters. We show that it is feasible to deterministically create bright sub-Poissonian states of light using a compact (cm-sized) integrated CDP device and, furthermore, reach other useful nonclassical output states. The generation of nonclassical light occurs in the regime when a conventional singlephoton loss is still not affecting significantly the process of the state generation.

The outline of the paper is as follows. Firstly, in Sec. II, we present the CDP scheme and describe the hierarchy of models, which can be used to account for the state dynamics. We reveal how the description of the whole network can be reduced to the description of just a single superposition mode ("single-mode model"). In Sec. III, we show that the regime of interest for us is the initial stage of the dynamics, when strong nonclassicality can be obtained. We develop an analytic model for evolution of the Mandel parameter and also show that at this stage the dynamics can be well captured by the linearization approach for the quantum correction to the field amplitude. The single-mode model allows us to present a simple and illustrative analysis of the PhoG-device feasibility providing necessary parameters of the setup, and ways to optimize it.

In Secs. IV and V we analyze dynamics of the system beyond the simplest single collective mode model. We pay special attention to the two-mode model (Sec. IV), which exhibits a number of nonclassical features not captured by the single-mode model, such as entanglement generation. After that in Sec. VI we proceed to the complete network showing that for the initial stage of dynamics the predictions made within the single-mode model are indeed true. 
Strong photon-number squeezing is shown to emerge for moderate lengths of the CDP circuit with realistic losses and nonlinearity. Also, to assess the impact of specific effects of short-pulse propagation in our system of coupled waveguides, we dynamically evolved the spectral and temporal properties of the pulse via the nonlinear Schrödinger equation, and analyzed their influence on the effectiveness of the NCL mechanism taking into account the combined effect of chromatic dispersion, self-phase modulation, and self-steepening of the pulse.

\section{HIERARCHY OF MODELS}

In the case of a large number of interacting quantum modes, a large number of photons and in the presence of nonlinearity, an exact description of the quantum-state dynamics of our system is a formidable task. To tackle the problem, we develop the hierarchical approach to model the photon gun and optimize its structure. The complete CDP scheme is reduced to much simpler systems of a few modes as shown in Fig. 2. These systems are much more tractable and can be analyzed, optimization can be carried out, approximations can be verified, and then the obtained results can be validated by the approximate numerical analysis of the complete CDP circuit.

The original scheme underlying all the subsequent discussion is depicted in Figs. 1(b) and 1(c): two waveguides $a$ and $b$ are coupled to the third waveguide $c_{0}$ (but not to each other), and this waveguide $c_{0}$ is coupled to the "tail" of next-neighbour coupled single-mode waveguides $c_{j}, j=1 \ldots N$. In our consideration, we assume identical single-mode waveguides described by the corresponding bosonic creation and annihilation operators. We assume that some initial state is created in the network, and then

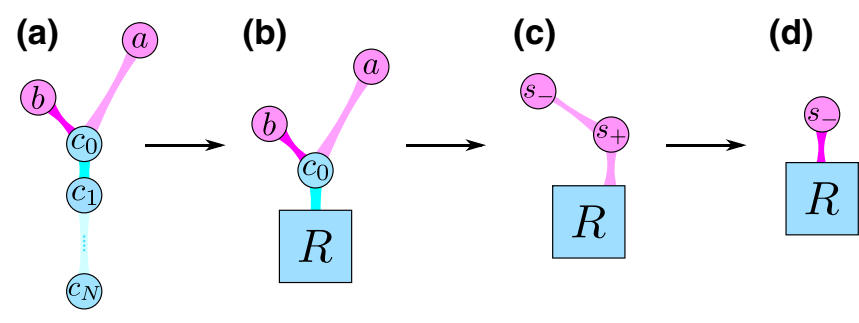

FIG. 2. Hierarchy of the PhoG models (a)-(d). The model (a) corresponds to the setup shown in Figs. 1(b) and 1(c) of coupled single-mode waveguides. Modes $a$ and $b$ interact with mode $c_{0}$ coupled also to the "tail" of modes $c_{1 \ldots N}$. The three-mode model (b) corresponds to the adiabatically eliminated "tail" of waveguides retaining only the first waveguide of the tail, which is highly lossy due to direct coupling to the reservoir $R$. This effectively corresponds to the system presented in Ref. [24]. The model (c) shows the "two-mode model," the model with two superposition, collective modes $s_{ \pm}$Eq. (5) obtained by eliminating the third mode $c_{0}$ of (b). Finally, the single-mode model (d) is obtained by adiabatic elimination of one of the collective modes in (c). this state evolves. Thus, the dynamics of this system is described by the following master equation for the density matrix $\rho$ :

$$
\frac{d}{d t} \rho=-i[H, \rho]+\gamma_{1}\left[\mathcal{L}(a)+\mathcal{L}(b)+\sum_{j=0}^{N} \mathcal{L}\left(c_{j}\right)\right] \rho,
$$

where the Lindbladians $\mathcal{L}$ describing common singlephoton loss with the rate $\gamma_{1}$ are given by $\mathcal{L}(x) y=x y x^{\dagger}-$ $\frac{1}{2} x^{\dagger} x y-\frac{1}{2} y x^{\dagger} x$ for operators $x$ and $y$; the density matrix for all modes is $\rho$ and the Hamiltonian $H=H^{\text {int }}+H^{\text {Kerr }}$ consists of two parts. The first part describes linear interactions between the modes

$$
H^{\mathrm{int}}=g_{a} a^{\dagger} c_{0}+g_{b} b^{\dagger} c_{0}+\sum_{j=1}^{N} g_{j} c_{j-1}^{\dagger} c_{j}+\text { h.c. }
$$

where $g_{a, b}$ are couplings of the modes $a, b$, respectively, to the third mode $c_{0}$, and $g_{j}$ is the coupling between mode $c_{j-1}$ and $c_{j}$. The part $H^{\text {Kerr }}$ describes the self-Kerr interaction of each mode,

$$
H^{\mathrm{Kerr}}=\frac{U}{2} \sum_{\forall x}\left(x^{\dagger}\right)^{2} x^{2}, \quad x=a, b, c_{j}, \quad j=0,1 \ldots N
$$

$U$ is the Kerr nonlinear interaction constant.

For a large number of waveguides $N$ and large initial number of photons, the complete model, Eqs. (1) and (2), is practically intractable without approximations. However, we are interested in the regime when the "tail" formed by the waveguides $c_{j}, j=1 \ldots N$, can be considered a dissipative reservoir rapidly guiding away field from the mode $c_{0}[14,15]$. Assuming that the tail functions as a Markovian reservoir with the decay rate $\gamma_{c}$, we arrive at the three-mode model depicted in Fig. 2(b) and described by the following master equation for the three-mode density matrix:

$$
\frac{d}{d t} \rho_{3}=-i\left[H_{3}, \rho_{3}\right]+\left[\gamma_{1} \mathcal{L}(a)+\gamma_{1} \mathcal{L}(b)+\gamma \mathcal{L}\left(c_{0}\right)\right] \rho_{3},
$$

where $\gamma=\gamma_{1}+\gamma_{c}$, the Hamiltonian is $H_{3}=H_{3}^{\text {int }}+H_{3}^{\text {Kerr }}$ with $H_{3}^{\text {Kerr }}$ as given in Eq. (3) and the interaction part is $H_{3}^{\text {int }}=g_{a} a^{\dagger} c_{0}+g_{b} b^{\dagger} c_{0}+$ h.c.

The three-mode model of Fig. 2(b) is still too complicated for exact analysis in the case of a large number of photons. Further simplification can be reached assuming that the decay rate into the "tail" $\gamma_{c}$ is large enough to allow for the adiabatic elimination of the mode $c_{0}$. Let us 
introduce symmetric and antisymmetric collective modes:

$$
s_{+}=\frac{1}{G}\left(g_{a} a+g_{b} b\right), \quad s_{-}=\frac{1}{G}\left(g_{a} b-g_{b} a\right),
$$

with $G=\sqrt{g_{a}^{2}+g_{b}^{2}}$ and coupling constants $g_{a, b}$ assumed real. Then adiabatic elimination of mode $c_{0}$ leads to the following two-mode master equation [24,25]:

$$
\frac{d}{d t} \rho_{2}=-i\left[H_{2}, \rho_{2}\right]+\left[\gamma_{1} \mathcal{L}\left(s_{-}\right)+\left(\Gamma+\gamma_{1}\right) \mathcal{L}\left(s_{+}\right)\right] \rho_{2},
$$

with $\Gamma=4 G^{2} / \gamma$. The Hamiltonian $H_{2}=H_{2}^{\text {int }}+H_{2}^{\text {self }}$ is given by

$$
\begin{aligned}
H_{2}^{\text {self }} & =\varsigma_{1}\left(n_{+}^{2}+n_{-}^{2}\right)+\varsigma_{2} n_{+} n_{-}+\varsigma_{3}\left(n_{+}+n_{-}\right), \\
H_{2}^{\text {int }} & =\varsigma_{4}\left(s_{+}^{\dagger} s_{-}\right)^{2}+\varsigma_{5} s_{+}^{\dagger} s_{-}\left(n_{-}-n_{+}-1\right)+\text { h.c. },
\end{aligned}
$$

where $n_{ \pm}=s_{ \pm}^{\dagger} s_{ \pm}$are the photon-number operators, and the coefficients read

$$
\begin{aligned}
& \varsigma_{1}=\frac{U}{2 G^{4}}\left(g_{a}^{4}+g_{b}^{4}\right), \quad \varsigma_{2}=\frac{4 U}{G^{4}}\left(g_{a} g_{b}\right)^{2}, \\
& \varsigma_{3}=\varsigma_{2} / 4-U / 2, \quad \varsigma_{4}=\varsigma_{2} / 4, \quad \varsigma_{5}=\frac{U}{G^{4}} g_{a} g_{b}\left(g_{a}^{2}-g_{b}^{2}\right) .
\end{aligned}
$$

The two-mode model is depicted in Fig. 2(c).

Finally, if the state of the superposition mode $s_{+}$decays to the vacuum much quicker than the typical timescale of the dynamics of the superposition mode $s_{-}$, one arrives to the single-mode model of Fig. 2(d) described by the following master equation [24,25]:

$$
\begin{aligned}
\frac{d}{d t} \rho_{1}= & {\left[\gamma_{1} \mathcal{L}\left(s_{-}\right)+\gamma_{2} \mathcal{L}\left(s_{-}^{2}\right)+\gamma_{3} \mathcal{L}\left(n_{-} s_{-}\right)\right] \rho_{1} } \\
& +-i\left[\varsigma_{1} n_{-}^{2}+\varsigma_{3} n_{-}, \rho_{1}\right]
\end{aligned}
$$

where the decay rates are given by

$$
\gamma_{2}=\frac{4 U^{2}\left(g_{a} g_{b}\right)^{4}}{G^{8}\left(\Gamma+\gamma_{1}\right)}, \quad \gamma_{3}=\frac{4 U^{2}\left(g_{a} g_{b}\right)^{2}}{G^{8}\left(\Gamma+\gamma_{1}\right)}\left(g_{a}^{2}-g_{b}^{2}\right)^{2} .
$$

The master equation (8) takes account of the three decay channels for the antisymmetric collective mode: conventional single-photon loss $\left(\gamma_{1}\right)$, two-photon loss $\left(\gamma_{2}\right)$, and $\operatorname{NCL}\left(\gamma_{3}\right)$. We refer to the decay described by the Lindblad operator $n_{-} s_{-}$as "nonlinear coherent loss" as the eigenstates of the operator $n_{-} s_{-}$are named "nonlinear coherent states" [27]. The Lindblad operator $n_{-} s_{-}$responsible for NCL can be considered as the annihilation operator of so-called $f$-deformed harmonic oscillator [26].

Note that the unitary part of the master equation, the last term in Eq. (8) connected to the self-Kerr interaction, does not manifest itself in the dynamics of the diagonal elements of the density matrix $\rho_{1}$.

The model Eq. (8) is much easier to tackle for a large number of photons than the two-mode model Eq. (6). Moreover, it allows us to obtain some general conclusions about nonclassicality, which are valid even for numbers of photons so large that an exact solution becomes unfeasible even for the single-mode model.

\section{SINGLE-MODE MODEL}

In this section we analyze the simplest single-mode scheme of Fig. 2(d) to describe the generation of the bright sub-Poissonian state from the semiclassical coherent input, and outline the way to optimize the scheme by choosing the amplitude of the initial coherent state and parameters of the PhoG for realistic CDP circuits with significant linear loss and moderate Kerr nonlinearity. We establish an existence of universal parameters $X, Y$, which are determined by effective nonlinearity and thus allow us to characterize the scheme performance even for realistic small optical nonlinearities and, consequently, very large numbers of photons, when solving the master equation becomes hardly possible. Finally, we demonstrate the practical feasibility of the scheme.

\section{A. Nonlinear loss dynamics}

There are two kinds of nonlinear loss present in the single-mode master equation (8). The first one is the twophoton loss described by the term $\mathcal{L}\left(s_{-}^{2}\right)$ and the second kind is the NCL described by the term $\mathcal{L}\left(n_{-} s_{-}\right)$. Both these kinds of losses are able to produce photon-number squeezing. However, their timescales and asymptotic states are different. The two-photon loss asymptotically leads to a mixture of single-photon and vacuum states $[28,29]$. NCL asymptotically leads to the single-photon state [24]. We show here that for the initial coherent state with large amplitude, $|\alpha| \gg 1$ and the assumed equal decay rates, $\gamma_{2}=\gamma_{3}$, NCL leads to much faster decay of the average photon number in the antisymmetric collective mode $s_{-}$ than the two-photon loss and thus to more rapid narrowing of the photon-number distribution in $s_{-}$.

The degree of photon-number distribution squeezing can be conveniently described by the Mandel parameter [30], which for the superposition mode $s_{-}$reads

$$
Q=\frac{\left\langle\left(s_{-}^{\dagger}\right)^{2} s_{-}^{2}\right\rangle}{\left\langle n_{-}\right\rangle}-\left\langle n_{-}\right\rangle
$$

Mandel parameter $Q=-1$ corresponds to the perfect squeezing (Fock states), zero is for coherent states. This parameter can be inferred from dynamics of only the diagonal elements of the density matrix Eq. (8) given by the 
following equation:

$$
\begin{aligned}
\frac{d}{d t} \rho_{n}= & -\left[\gamma_{1} n+\gamma_{2} n(n-1)+\gamma_{3} n(n-1)^{2}\right] \rho_{n} \\
& +\left[\gamma_{1}(n+1)+\gamma_{3}(n+1) n^{2}\right] \rho_{n+1} \\
& +\gamma_{2}(n+1)(n+2) \rho_{n+2},
\end{aligned}
$$

where $\left\langle n\left|\rho_{1}(t)\right| n\right\rangle=\rho_{n}$ are the density-matrix elements in the Fock-state basis.

Note that for the symmetric case, $g_{a}=g_{b}$, the NCL decay rate $\gamma_{3}$ is zero. For asymmetric coupling, $g_{a} \neq g_{b}$, we fix the parameter $g_{a}$ and start varying the parameter $x=g_{b} / g_{a}$ for $x \in[0,1]$. The maximal value of the rate $\gamma_{3}$ is then given by the condition $d \gamma_{3}(x) / d x=0$ and leads to the following result for the ratio of the interaction constants to maximize NCL:

$$
\left(g_{b} / g_{a}\right)_{\mathrm{opt}}=\sqrt{2}-1 .
$$

For this optimal coupling ratio, we have

$$
\gamma_{3}=\frac{U^{2}}{4\left(\gamma_{1}+\Gamma\right)}
$$

In further considerations, we adopt this ratio for estimations.

In Fig. 3 examples of the Mandel parameters and average photon-number dynamics are given for different numbers of photons (several hundred) of the initial coherent state. Fig. 3 reveals a number of important features in the dynamics of the antisymmetric collective mode:

1. The Mandel parameter rapidly decreases at the initial stage of the dynamics. Only a small percentage of photons is lost when quite considerable negative values of the Mandel parameter are reached [less than $15 \%$ to have significant photon-number squeezing with $Q<-0.25$, see Figs. 3(c) and 3(d)].

2. The practically reachable values of the Mandel parameter are approximately limited to $Q=-0.8$ (see also Ref. [31]). This minimal value is shifted to smaller interaction length by increasing the average number of photons in the initial state.

3. Dynamics due to NCL occurs much faster than dynamics due to the two-photon loss. As seen in Fig. 3(a), even a hundredfold increase in the two-photon decay rate, $\gamma_{2}$, does not influence much the initial stage of the $Q$ parameter dynamics. In general, the ratio between these two timescales is determined by the ratio between the coupling constants, $g_{b} / g_{a}: \gamma_{3}=\gamma_{2}\left[1-\left(g_{b} / g_{a}\right)^{2}\right] /\left(g_{b} / g_{a}\right)^{2}$ [see Eq. (9) and text around Eq. (12)].

4. NCL can outrun linear loss. Figure 3(b) shows that even large linear loss weakly affects the initial stage of the dynamics.
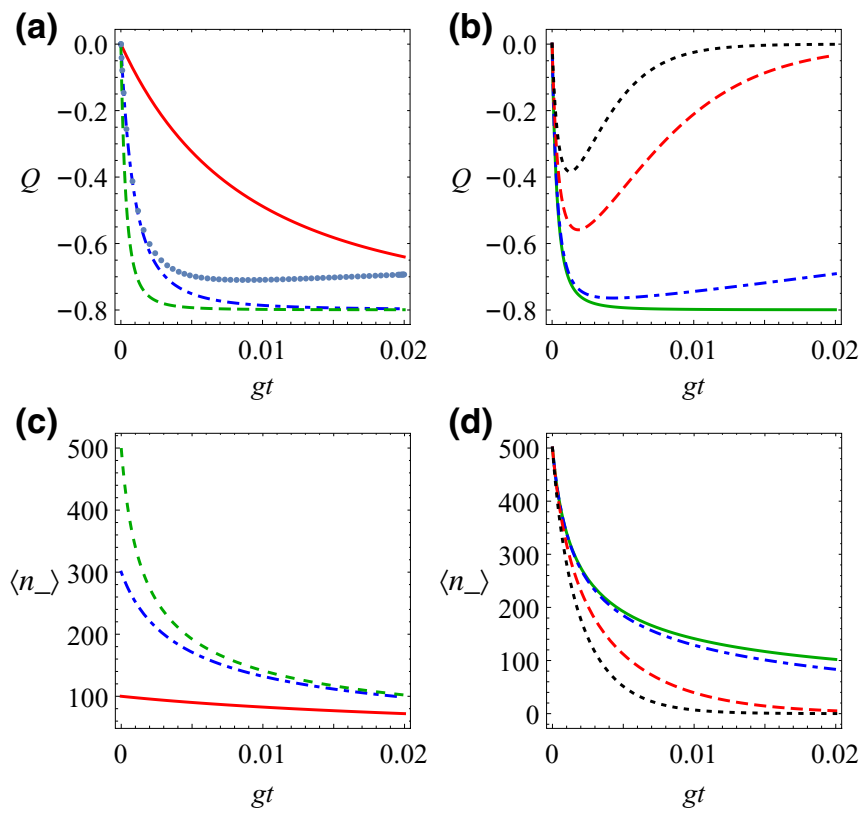

FIG. 3. Mandel parameter $Q$ (a) and the average number of photons (c) for different values of the initial number of photons of the input coherent state and no linear loss, $\gamma_{1}=0$. The solid, dotdashed, and dashed lines correspond to $\left\langle n_{-}(0)\right\rangle=100,300,500$, respectively. The line marked with round dots in (a) shows the Mandel parameter for $\left\langle n_{-}(0)\right\rangle=300$ and $\gamma_{2}$ increased 100 times (enhanced two-photon loss) compared with that given by Eq. (9). Mandel parameter $Q$ (b) and the average number of photons (d) for different values of linear loss rate $\gamma_{1}$ and the initial coherent state with the average number of photons $\left\langle n_{-}(0)\right\rangle=500$. The solid, dot-dashed, dashed, and dotted lines correspond to $\gamma_{1}=0,20 g, 200 g, 400 g$, respectively. The scaling parameter $g$ here and in the subsequent simulations is taken to be a half of the third-order nonlinear rate, $U=2 g$. For all the panels, the optimal ratio Eq. (12) is taken. Also, it is assumed that the symmetric mode loss rate $\Gamma=432 \mathrm{~g}$.

5. Consider now the case of no linear loss. For different large initial numbers of photons, after some time the average numbers of photons tends to largely the same stationary value [Fig. 3(c)]. It is a signature behavior of NCL [24].

\section{B. Universal parameters}

As we show, the two-photon loss only weakly influences the dynamics of photon-number squeezing. Also, large photon-number squeezing is generated at the initial stage of dynamics when only a relatively small number of photons is lost. These two facts allow us to introduce two dimensionless parameters allowing an estimation of the feasibility of the scheme. These two parameters are

$$
X=\gamma_{3}\left[\left\langle n_{-}(0)\right\rangle\right]^{2} t_{\text {fix }}, \quad Y=\frac{\gamma_{1}}{\gamma_{3}\left\langle n_{-}(0)\right\rangle^{2}},
$$

where $t_{\text {fix }}$ is the fixed interaction time (defined by the length of the PhoG). The parameter $X$ defines the 

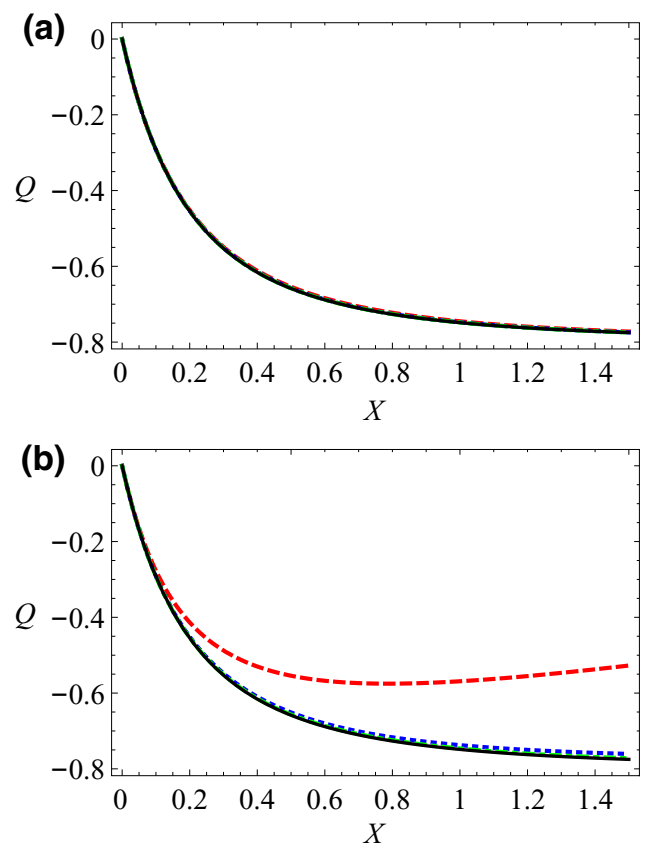

FIG. 4. Dependence of the Mandel parameter $Q$ on the parameter $X$ of Eq. (14) for different initial average numbers of photons $\left\langle n_{-}(0)\right\rangle$ in the absence of linear loss, $\gamma_{1}=0$, (a) and for significant linear loss, $\gamma_{1}=10 \mathrm{~g}$, (b). Dashed, dotted, and dotdashed lines are obtained by solving Eq. (11) and correspond to $\left\langle n_{-}(0)\right\rangle=100,400,900$, respectively. Solid line corresponds to the analytical approximation given by Eq. (17). Other parameters are the same as for Fig. 3. The lines merge together and are almost indistinguishable for $\gamma_{1}=0$. The line for $\left\langle n_{-}(0)\right\rangle=900$ merges with approximate solution (17) even for $\gamma_{1}=10 \mathrm{~g}$.

reachable value of the Mandel parameter, $Q$, in the absence of the linear and two-photon loss, and the parameter $Y$ defines the tolerable level of the linear loss when the value of $Q$ is still defined by $X$.

In terms of parameter $Y$, one has to have $Y<1$ for optimal $Q$ for a given $X$, Fig. 4(a). Of course, even larger values of $Y$ still allow for considerable $Q$. However for that, larger values of $X$ are required.

Such a dependence of photon-number squeezing on the universal parameters can be explained by simple analytic considerations. For a large number of photons of the initial coherent state, the initial photon number distribution can be with high precision described by the Gaussian. Let us assume that for the initial stage of the dynamics the distribution can still be described by the Gaussian

$$
\rho_{n}(t)=\frac{1}{\sqrt{2 \pi} \sigma(t)} \exp \left\{-\frac{\left[n-\left\langle n_{-}(t)\right\rangle\right]^{2}}{2 \sigma(t)^{2}}\right\}
$$

where $\sigma(t)=[Q(t)+1]\left\langle n_{-}(t)\right\rangle$. In Appendix $\mathrm{A}$ it is shown that Gaussianity of the photon-number distribution holds very well for $\left\langle n_{-}(t)\right\rangle \gg 1$. Assumption of a smooth photon-number distribution slowly changing with $n$ [like the one given by Eq. (15) for large $\left.\left\langle n_{-}(0)\right\rangle\right]$ allows us to introduce a continuous variable $n$, to make an approximation $\rho_{n+m} \approx \rho_{n}+m(d / d n) \rho_{n}+m^{2} \frac{1}{2}\left(d^{2} / d n^{2}\right) \rho_{n}$, and to calculate averages as

$$
\left\langle n_{-}(t)^{m}\right\rangle=\int_{0}^{+\infty} d n \rho_{n} n^{m} .
$$

Thus, one can obtain the following equation for the Mandel parameter:

$$
\frac{d}{d t} Q \approx-\gamma_{1} Q-2 \gamma_{2}\left\langle n_{-}\right\rangle(3 Q+1)-\gamma_{3}\left\langle n_{-}\right\rangle^{2}(5 Q+4) .
$$

Neglecting linear and two-photon losses, in the continuous approximation for Eq. (11) one obtains that $\left\langle n_{-}(X)\right\rangle \approx$ $\left\langle n_{-}(0)\right\rangle / \sqrt{1+2 X}$ (see Appendix A). For the Mandel parameter we get

$$
Q(X) \approx-\frac{4}{5}\left[\frac{1}{(1+2 X)^{5 / 2}}-1\right] .
$$

This situation is illustrated in Fig. 4. Figure 4(a) shows that in the absence of linear loss neither the initial number of photons nor the interaction time by themselves are the parameters defining possible $Q$. Practically, it depends only on $X$. The black solid line in Fig. 4(a) shows the result provided by approximation (17) for several initial states with hundreds of photons. It is obvious that Eq. (17) very closely reproduces the exact solution of Eq. (11). Equation (11) also shows why the influence of two-photon losses is so small for $\gamma_{2}$ close to $\gamma_{3}$ : the timescales are determined by $\propto \gamma_{2} n^{2}$ for two-photon loss and $\propto \gamma_{3} n^{3}$ for NCL. For large photon numbers and $\gamma_{2} \approx \gamma_{3}$, the NCL obviously dominates.

Figure 4(b) shows the influence of linear loss (described by the parameter $Y$ ) on the reachable value of the Mandel parameter. For a given linear loss of $\gamma_{1}=10 \mathrm{~g}$, for $\langle n(0)\rangle=900$ one has $Y<1$, and the curve describing the achievable values of the Mandel parameter (dash-dotted line) is practically coinciding with the approximation given by Eq. (17). For small $Y$ any influence of the linear loss is indeed small. For a smaller initial number of photons, $\langle n(0)\rangle=100$, when $Y>1$, one has considerable difference with analytic predictions [dashed line in Fig. 4(b)].

\section{Feasibility}

Universal parameters $X$ and $Y$ are decisive to ensure the optimal performance of PhoG as a deterministic source of sub-Poissonian light. For PhoG design we have a "rule-ofthumb": we should aim to make $X$ as large as possible, and $Y$ as small as possible. In this section we demonstrate that it is feasible to reach $X>0.3$ and $Y<1$ for realistic waveguide structures. 
First of all, we connect the nonlinear interaction constant $U$ in expressions (9) with quantities commonly used for description of the Kerr nonlinearity in waveguide structures. We assume that the pulse of the finite length propagates through the waveguide, and the pulse length $L_{\text {eff }}$ is much smaller than the waveguide length; we introduce the propagation length as $z=c t_{\mathrm{fix}} / n_{\mathrm{eff}}$, where $n_{\mathrm{eff}}$ is the effective refractive index of the waveguide mode. Thus, the nonlinear interaction constant in our model can be written in the following way (see, for example, Ref. [32]):

$$
U=2 \hbar \omega \frac{\omega}{V_{\mathrm{eff}}} \frac{n_{2}}{n_{\mathrm{eff}}},
$$

where $n_{2}$ is the nonlinear refractive index and $V_{\text {eff }}$ is the mode volume. For our model, we assume $V_{\text {eff }} \approx A_{\text {eff }} L_{\text {eff }}$, where $A_{\text {eff }}$ is the effective area. The effective area is defined as

$$
A_{\mathrm{eff}}^{-1}=\iint d^{2} \vec{s}|E(\vec{s})|^{4},
$$

where $E(\vec{s})$ is the normalized transversal field profile,

$$
\iint d^{2} \vec{s}|E(\vec{s})|^{2}=1 .
$$

Here we do not consider the influence of losses on the nonlinear interaction constant [33].

In a fiberlike waveguide it is customary to introduce the nonlinear fiber coefficient:

$$
\bar{\gamma}^{\mathrm{NL}}=\frac{\omega}{c} \frac{n_{2}}{A_{\mathrm{eff}}} \Rightarrow U=2 \frac{\hbar \omega}{T_{\mathrm{eff}} n_{\mathrm{eff}}} \bar{\gamma}^{\mathrm{NL}},
$$

where $T_{\text {eff }}$ is the pulse time duration in vacuum.

Now let us consider several waveguide arrangement with realistic parameters.

\section{Large waveguides in bulk glass}

Let us take coupling parameters as used in the CDP circuits in Ref. [15], $g_{a} \approx 200 \mathrm{~m}^{-1}$. The decay rate of the $c_{0}$ mode Fig. 2 can be approximated by $\gamma_{c} \approx 4 \sqrt{g_{a}^{2}+g_{b}^{2}}$, which for optimal coupling ratio results in $\Gamma \approx 216.5 \mathrm{~m}^{-1}$ (decay rate for the symmetric collective mode $s_{+}$, Fig. 2). For intended linear loss of about $0.5 \mathrm{~dB} / \mathrm{cm}$, we have $\gamma_{1} \approx 11.5 \mathrm{~m}^{-1}$, and $\gamma_{3} \approx 0.0011 \mathrm{~m}^{-1} \times U^{2}$. The dimensions of the waveguides as used in CDP circuits in Ref. [15] are approximately $4 \times 4 \mu \mathrm{m}^{2}$. For a conservative estimate, let us assume an effective modal area an order of magnitude larger than typical for single-mode fibers with similar core dimensions around $1000 \mathrm{~nm}$ (see, for example, Corning ${ }^{\circledR}$ HI 1060 fiber with the mode-field diameter of about $6.2 \mu \mathrm{m}$ at $1060 \mathrm{~nm}$ ), $A_{\text {eff }}=300 \mu \mathrm{m}^{2}$ (which would be more than three times larger even for typical modal areas at $1550 \mathrm{~nm}$ ). Thus, taking $n_{2}=3 \times 10^{-18} \mathrm{~W}^{-1} \mathrm{~m}^{2}$ and $n_{\text {eff }}=2.59$ typical for IG2 glass [34], for a $100-\mathrm{fs}$ pulse at $1060 \mathrm{~nm}$ we have $\gamma_{3} \approx 8 \times 10^{-18} \mathrm{~m}^{-1}$. Condition $Y=1$ of Eq. (14) requires energy levels of $1.2 \times 10^{9}$ photons per pulse or $224 \mathrm{pJ}$ per pulse. The feasibility of these energy levels in the context of our setup is confirmed by the recent work [35], where femtosecond pulses with energies more than $10 \mathrm{~nJ}$ at $1030 \mathrm{~nm}$ have been used for writing waveguides. Using all these parameters, for a $3-\mathrm{cm}$ waveguide we have $X \approx 0.33$, which is sufficient to reach a high degree of photon-number squeezing $Q<-0.5$. As follows from condition (14), for lower effective modal area one would need proportionally lower energies per pulse. For $A_{\text {eff }}=30 \mu \mathrm{m}^{2}$ only $25 \mathrm{pJ}$ per pulse is sufficient.

\section{Fiberlike systems}

Now let us use for the PhoG setup the parameters from the recent fiber-based interferometric scheme [36] for sub-Poissonian light generation. For the used polarization maintaining fiber (Nufern, HP-780) one has $\bar{\gamma}^{\mathrm{NL}}=8.51 \times$ $10^{-3} \mathrm{~W}^{-1} \mathrm{~m}^{-1}$ for $808 \mathrm{~nm}$. Using $n_{\text {eff }}=1.45$, we arrive at $\gamma_{3} \approx 9.2 \times 10^{-19} \mathrm{~m}^{-1}$. It is nearly an order of magnitude lower than for the bulk waveguides. However, much lower loss of $3.5 \mathrm{~dB} / \mathrm{km}$ implies that condition (14) is easier to satisfy. For that, $3 \times 10^{7}$ photons per pulse (or less than $10 \mathrm{pJ}$ per pulse) are needed. If $2 \times 10^{9}$ photons per pulse are used, $10 \mathrm{~cm}$ of the fiber are required to reach $X=0.37$ and squeezing of about $Q<-0.5$ (which is more or less in agreement with results obtained in Ref. [36]).

\section{Silicon nanowires}

Finally, let us consider the silicon nanowire arrangement as a possible platform for PhoG generators. Si-nanowire waveguides have typically very high nonlinearity and loss, and small lengths. We assume loss of $5 \mathrm{~dB} / \mathrm{cm}$ and moderate nonlinearity, $\bar{\gamma}^{\mathrm{NL}}=300 \mathrm{~W}^{-1} \mathrm{~m}^{-1}$ with other parameters being the same as above [37]. Notice that even for such loss level, $\Gamma$ is larger than $\gamma_{1}$, where $\gamma_{1} \approx 115 \mathrm{~m}^{-1}$. For $n_{\text {eff }}=3.5$ and wavelength of $1064 \mathrm{~nm}$, we obtain $\gamma_{3} \approx$ $7.75 \times 10^{-11} \mathrm{~m}^{-1}$, which is much larger than for all the previously considered cases. To satisfy $Y=1$, we require only $1.3 \times 10^{6}$ photons per pulse or less than $0.3 \mathrm{pJ}$ pulse energy. For this number of photons a $3-\mathrm{mm}$ waveguide would be sufficient to achieve $X=0.345$ and $Q<-0.5$.

\section{Linearization}

Here we demonstrate that, over the timescales when large photon-number squeezing occurs, at the initial stage of dynamics the evolution of the Mandel parameter can be adequately described using a linearization approach. This approximation is typical for consideration of nonlinear waveguide systems. Essentially, it is a linearization on a quantum correction to the creation and annihilation 
operators, $s_{-}=S_{-}+\delta s_{-}$, where the quantity $S_{-}=\left\langle s_{-}\right\rangle$is the classical amplitude, $\delta s_{-}$are the quantum fluctuations, which obey the same commutator as $s_{-}$, and $\left\langle\delta s_{-}\right\rangle=0$. The resulting system of equations allows us to find quantities $\left\langle s_{-}\right\rangle,\left\langle s_{-}^{\dagger}\right\rangle,\left\langle s_{-}^{2}\right\rangle,\left\langle s_{-}^{\dagger} s_{-}\right\rangle$, and $\left\langle s_{-}^{\dagger 2}\right\rangle$ and circumvents the computational complexity required in the case of exact solution for large photon numbers in a large number of modes.

Our starting point is Eq. (8), from which we may derive the following system of coupled equations:

$$
\begin{aligned}
\partial_{t}\left\langle s_{-}\right\rangle & =c_{1}\left\langle s_{-}\right\rangle+c_{2}\left\langle s_{-}^{\dagger} s_{-}^{2}\right\rangle-\frac{\gamma_{3}}{2}\left\langle s_{-}^{\dagger 2} s_{-}^{3}\right\rangle, \\
\partial_{t}\left\langle s_{-}^{\dagger}\right\rangle & =c_{1}^{*}\left\langle s_{-}^{\dagger}\right\rangle+c_{2}^{*}\left\langle s_{-}^{\dagger 2} s_{-}\right\rangle-\frac{\gamma_{3}}{2}\left\langle s_{-}^{\dagger 3} s_{-}^{2}\right\rangle, \\
\partial_{t}\left\langle s_{-}^{2}\right\rangle & =c_{3}\left\langle s_{-}^{2}\right\rangle+c_{4}\left\langle s_{-}^{\dagger} s_{-}^{3}\right\rangle-\gamma_{3}\left\langle s_{-}^{\dagger 2} s_{-}^{4}\right\rangle, \\
\partial_{t}\left\langle s_{-}^{\dagger 2}\right\rangle & =c_{3}^{*}\left\langle s_{-}^{\dagger 2}\right\rangle+c_{4}^{*}\left\langle s_{-}^{\dagger 3} s_{-}\right\rangle-\gamma_{3}\left\langle s_{-}^{\dagger 4} s_{-}^{2}\right\rangle, \\
\partial_{t}\left\langle s_{-}^{\dagger} s_{-}\right\rangle & =-\gamma_{1}\left\langle s_{-}^{\dagger} s_{-}\right\rangle+c_{5}\left\langle s_{-}^{\dagger 2} s_{-}^{2}\right\rangle-\gamma_{3}\left\langle s_{-}^{\dagger 3} s_{-}^{3}\right\rangle,
\end{aligned}
$$

with coefficients $c_{1}=\left(-\gamma_{1} / 2+i \varsigma_{1}+i \varsigma_{3}\right), c_{2}=\left(\gamma_{2}-\right.$ $\left.\gamma_{3}+2 i \varsigma_{1}\right), \quad c_{3}=\left(-\gamma_{1}-\gamma_{2}-\gamma_{3}+4 i \varsigma_{1}+2 i \varsigma_{3}\right), \quad c_{4}=$ $\left(-2 \gamma_{2}-5 \gamma_{3}+4 i \varsigma_{1}\right)$, and $c_{5}=\left(-2 \gamma_{2}-\gamma_{3}\right)$.

This system of equations is not closed, and so cannot yet be solved. To proceed, we must perform the linearization procedure in order to reduce operator products in Eq. (21) to at most second order, thereby closing the system of equations and allowing us to obtain its solution. The linearization can be done in a standard manner using the cumulant expansion (see Appendix B). The linearized forms of Eqs. (21) are excplitly shown in Appendix C.

The solution of the linearized forms of Eq. (21) are used to approximate $Q$, and in Fig. (5) this result is compared to the exact method from the previous section. The linearization approximation (dashed lines) accurately predicts the evolution of $Q$ over the initial stages of evolution, and actually underestimates $|Q|$ in the later stages, although including realistic $\gamma_{1}$ allows the approximation to remain accurate as the nonclassical output state is pushed towards the vacuum.

Since when $\gamma_{1}=0$ the linearization approximation remains accurate over the timescales of interest [see Fig. 5(a)], and since the presence of realistic loss makes the approximation increasingly accurate [see Fig. 5(b)], we may confidently apply the linearization approach over the parameter regimes of physical interest - short times and realistic loss - even in the case where a fully quantum treatment would be intractable, i.e., large $\langle n(0)\rangle$ or large number of modes.

\section{THE SYMMETRIC TWO-MODE MODEL}

In the previous section, we show that two-photon loss is far less efficient in producing photon-number squeezing
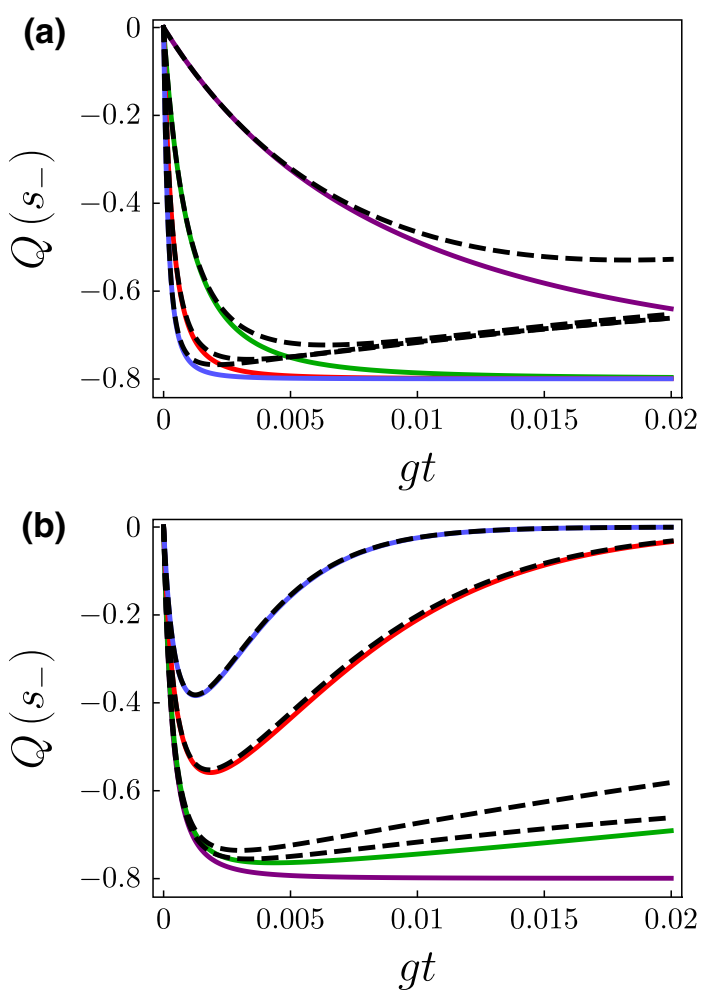

FIG. 5. Evolution of the Mandel $Q$ parameter is accurately predicted by the linearization method over the initial stages of evolution, and $|Q|$ is underestimated at later stages. Dashed, linearized. Solid, exact, as in Fig. 3. $U=2 g$ and $\Gamma=432 g$. (a) Initial photon number $\left\langle n_{-}(0)\right\rangle=100,300,500,700$ (top to bottom), with $\gamma_{1}=0$. (b) $\left\langle n_{-}(0)\right\rangle=500, \gamma_{1}=0,20 g, 200 g, 400 g$ (bottom to top). With realistic linear loss rates $\gamma_{1}$ our approximation remains accurate even in the late stages of evolution. Both graphs use the same time scaling $g t$ as Fig. 3 .

than NCL. However, it is worth pointing out that even symmetric system exhibiting only two-photon loss can be of use. To show it, let us recourse to the two-mode model of Fig. 2(c). First of all, even a symmetric system is able to produce entangled states of waveguide modes asymptotically, when the state of the symmetric superposition mode $s_{+}$is the vacuum (Sec. IV A). The second and rather nontrivial feature of the symmetric system is the possibility of producing few-photon entangled states by driving the rapidly decaying collective mode $s_{+}$(Sec. IV B). This scheme can also be used for probabilistic generating of single photons.

\section{A. Modal entanglement}

Generally, the state produced by the two-photon loss is quite far from Gaussian (asymptotically, the initial bright coherent state is driven by the two-photon loss toward the superposition of the vacuum and single-photon state [28, $38]$ ). However, one can still obtain a lower bound on the entanglement between modes from the covariance matrix 


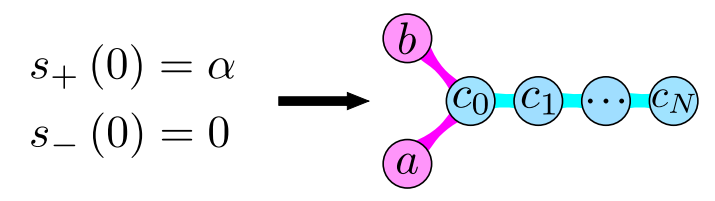

FIG. 6. Two-mode PhoG model: symmetric CDP circuit with initial excitation in symmetric collective mode $s_{+}$.

for modes $a, b: \sigma_{k l}=\frac{1}{2}\left\langle d_{k} d_{l}+d_{l} d_{k}\right\rangle-\left\langle d_{k}\right\rangle\left\langle d_{l}\right\rangle$, with the vector $\vec{d}=(1 / \sqrt{2})\left[a+a^{\dagger}, i\left(a^{\dagger}-a\right), b+b^{\dagger}, i\left(b^{\dagger}-b\right)\right]$ $[39,40]$. This bound is provided by the (Gaussian) logarithmic negativity $\mathcal{N}[39,40]$, which quantifies violation of the positive partial transpose criterion [41]. The logarithmic negativity $\mathcal{N}$ is defined as $\mathcal{N}=\max \{0,-\log \lambda\}$, where $\lambda$ is the smallest symplectic eigenvalue of the partially transposed matrix $\sigma_{k l}$. Elements of this matrix are provided by the linearized approach considered in the previous section.

For example, for $\left\langle a^{\dagger} a(0)\right\rangle=\left\langle b^{\dagger} b(0)\right\rangle=2500, U=2 g$, $\gamma_{c}=15 g, \gamma_{1}=11.5 \mathrm{~g}$ and symmetric coupling $g_{a}=g_{b}=$ $60 g$, the system evolves to logarithmic negativities $\mathcal{N} \approx$ 1.25 between modes $a$ and $b$ within $0.01 g t$, while modes $a$ and $b$ each contain 18 photons. Thus symmetric PhoG generates entanglement between modes $a$ and $b$ (though is unable to produce large photon-number squeezing in the initial stages of the dynamics).

\section{B. Photon-pair generation}

Despite the quick decay of the symmetric mode $s_{+}$, it still can create a nonclassical state in the mode $s_{-}$. Indeed, for the symmetric case of Fig. $6, g_{a}=g_{b} \equiv g$, we have $\varsigma_{5}=0$ and the Hamiltonian part describing interaction between superposition modes is

$$
H_{2}^{\text {int }}=\varsigma_{4}\left(s_{+}^{\dagger} s_{-}\right)^{2}+\text { h.c. }
$$

The Hamiltonian $H_{2}^{\text {int }}$ for the case describes the four-wavemixing process of transferring two photons of initially excited decaying mode $s_{+}$to the initially empty mode $s_{-}$. Thus the symmetric PhoG of Fig. 6 can be designed to reproduce the four-wave-mixing process, which is known to allow for the generation of entangled photon pairs $[42,43]$. But notice that in our case there is no problem of separating the generated state from the pump. For sufficiently long PhoG the driving excitation of the mode $s_{+}$ completely decays leaving photons only in the mode $s_{-}$.

The process of the pair-generation dynamics with weak coherent initial states is illustrated in Fig. 7 with the photon-number distribution of mode $a$. Initial coherent states with equal amplitudes are excited in waveguides $a$ and $b$ (corresponding to $s_{+}, s_{-}$as shown in Fig. 6). For small propagation lengths, the remnant of the initial excitation dominates [Fig. 7(a)]. However, it quickly decays and

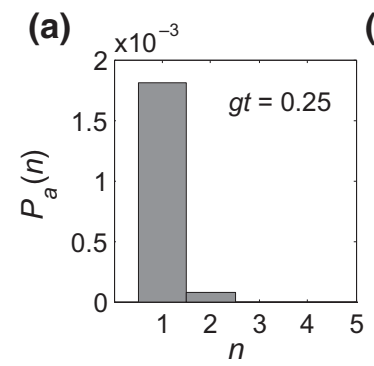

(b) $\times 10^{-4}$

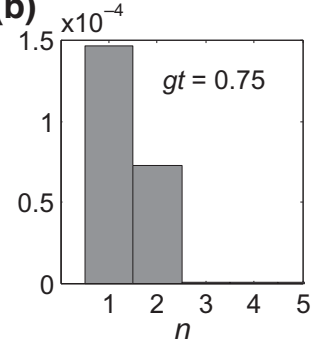

FIG. 7. $\quad P(n)$, the $n$-photon probabilities, in mode $a$ for different propagation times. The symmetric PhoG is taken, $g_{a}=g_{b}=g$. Weak coherent states with amplitudes $\alpha_{a}=0.5 ; \alpha_{b}=0.5$ are assumed as initial states of modes $a$ and $b$. Here zero-photon components are not shown. Notice that here the scaling constant $g$ is taken to be equal to the Kerr nonlinear constant, $U=g$; the loss in the collective symmetric mode is $\gamma_{c}=5 \mathrm{~g}$, and the linear loss rate is zero.

for larger times predominantly two photons in the antisymmetric mode are observed [Fig. 7(b)]. Just two photons in this mode correspond to the following entangled superposition of photons in waveguides $a$ and $b$ at the output of the PhoG:

$$
\begin{aligned}
\left|s_{-}\right\rangle^{\text {out }}\left|s_{+}\right\rangle^{\text {out }} & =|2\rangle_{-}|0\rangle_{+} \\
& =\frac{1}{2}\left(|2\rangle_{a}|0\rangle_{b}+|0\rangle_{a}|2\rangle_{b}-\sqrt{2}|1\rangle_{a}|1\rangle_{b}\right) .
\end{aligned}
$$

One can see the photon-number distribution corresponding to single- and two-photon part of the generated state (23) in Fig. 7(b). The ratio of the single-photon component to the two-photon component is approximately 2.05 for $g t=0.75$. Notice that by increasing amplitudes of the initial states, a squeezed vacuum state is created in mode $s_{-}$.

Another important difference with the conventional four-wave-mixing pair-generating schemes $[42,43]$ is that here we have the two entangled photons in two spatially separated modes. As can be seen in Ref. [15], at the PhoG output, the spatial distance between the modes exceeds considerably the mode cross section.

\section{THREE-MODE MODEL}

As seen in the previous sections, analysis of the singlemode model allows us to give simple and straightforward prescriptions for the practical realizations of the PhoG. The question is how close predictions given by the single-mode model approach the results for more complicated modal arrangements.

In this section, we analyze dynamics of a three-mode PhoG schematically shown in Fig. 2(c). Considering this model, we have in mind two purposes. Firstly, the threemode scheme can be realized in practice as it is, i.e., by 
arranging a strong loss in the waveguide $c_{0}$. It is feasible, for example, in semiconductor planar waveguide structures [44]. Secondly, the three-mode model still allows for a comparison between the exact solution and the singlemode approximation for a moderately large numbers of photons (few tens). One could also use it to verify the linearization approximation, and check the optimal ratio of the interaction constants $g_{b} / g_{a}$ for minimizing the Mandel parameter with a fixed interaction time and length.

To verify the transition from the three-mode model Eq. (4) to the single-mode model Eq. (8), the evolution of the mean photon number $\left\langle n_{-}(t)\right\rangle$ and the Mandel parameter $Q_{-}(t)$ of the antisymmetric collective mode $s_{-}$is simulated using the three-mode model and quantum trajectories approach [45]. Obtained results are then compared with the same quantities computed using the single-mode model with parameters taken from Eqs. (9). Figure 8 illustrates how close these models are for the optimal ratio $g_{b} / g_{a}$ Eq. (12) and large enough losses in the third, lossy, mode. Solid lines in the figure correspond to the three-mode model and dots correspond to the single-mode model. The figure is obtained for $g_{b} / g_{a}=\sqrt{2}-1, U=0.012 g_{b}, \gamma_{c}=$ $6.04 g_{b}, \gamma_{1}=0$.

Thus, the three-mode model confirms the main evolution characteristic stemming from the single-mode analysis, namely, rapid photon-number squeezing for relatively
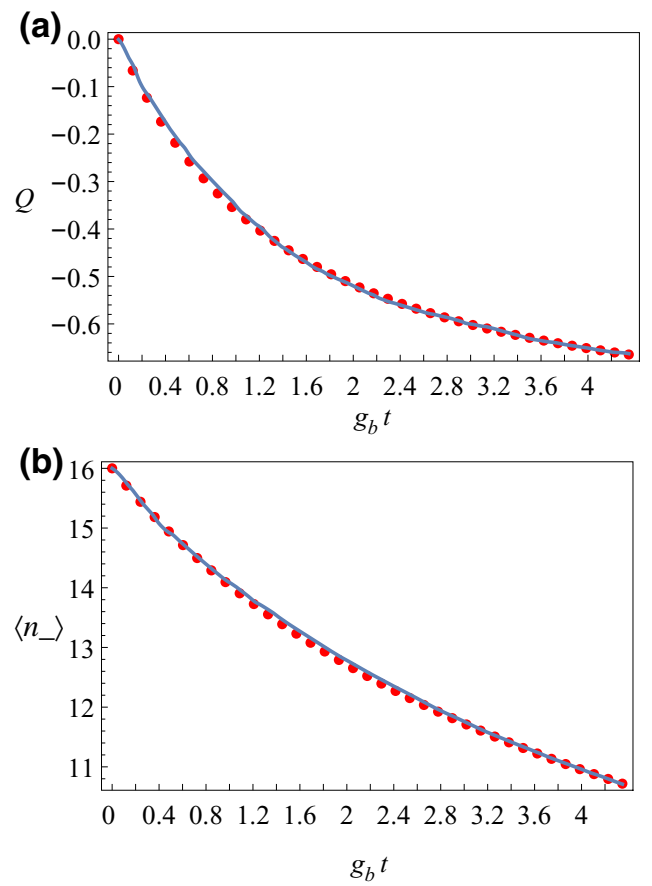

FIG. 8. Evolution of Mandel parameter (a) and mean photon number (b) of the antisymmetric mode $s_{-}$computed using the three-mode model (solid lines) and the single-mode model (dots) for the following parameters: $g_{b} / g_{a}=\sqrt{2}-1, U=0.012 g_{b}$, $\gamma_{c}=6.04 g_{b}, \gamma_{1}=0$. small decrease in photon number in the initial stage of evolution.

\section{MULTIMODE MODEL}

Finally, we consider a complete multimode model depicted in Fig. 2(a) for the realistic parameters described in Sec. III, and show that predictions made in that section still hold true for a multimode circuit of dissipatively coupled bosonic modes. We consider the multimode PhoG device with the two signal modes coupled to a long tail, which is described by Eq. (1).

We take the physical parameters from Sec. III C 1 for coupled waveguides in bulk glass, and the number of tail modes to be $N=28$. Since the Kerr-nonlinearity parameter is small, $U \sim 10^{-8}$, in order to reach the regime of strongly sub-Poissonian light we have to take a bright input coherent state containing $\mathcal{O}\left(10^{9}\right)$ photons. To model such large numbers of photons and long tail lengths we recourse to the linearization method described in Sec. III D along the lines demonstrated in Appendix B.

This linearization technique is applied to the full master equation, Eq. (1). Since in the multimode model the Lindblad operators are only first order in $a, b$, and $c_{j}$, only the third- and fourth-order linearization approximations are required. Therefore, we may reasonably expect the approximation to be more accurate in the multimode case than in the single-mode case, where linearization on sixth-order expectation values is required. We thus derive a closed system of equations for expectations up to second order. An example of the evolution of both $Q$ and $\left\langle n_{-}\right\rangle$is shown in Fig. 9.

When a coherent state containing $1.2 \times 10^{9}$ photons is initialized in mode $s_{-}$(solid red lines in Fig. 9), the system quickly evolves to a strongly sub-Poissonian state, even in the presence of a realistic loss rate $\gamma_{1}$. A brighter initial state results in larger $|Q|$ over shorter timescales, and in greater robustness of the sub-Poissonian output to linear loss $\gamma_{1}$.

For no linear loss, $\gamma_{1}=0$ (dashed line in Fig. 9(b)), $Q \approx$ -0.8 remains steady until a later time, which is proportional to $N$. Thus, the tail effectively acts as a Markovian reservoir, which justifies its adiabatic elimination in Eq. (4) [15]. Crucially, therefore, the long tail of modes combined with the self-Kerr interaction corresponds to the effects of nonlinear coherent loss into a Markovian reservoir (compare Figs. 3 and 9), and we note that the timescales over which this correspondence holds may be increased by increasing the tail length $N$.

Since $s_{-}$is a linear combination of modes $a$ and $b$, Eq. (5), the input coherent state with amplitude $\alpha$ into $s_{-}$ may be generated by initializing modes $a$ and $b$ with coherent states of appropriate amplitude. Alternatively, we may consider the initial condition $\langle a\rangle=\alpha$ and all other modes as vacuum. In this case, the behavior of $s_{-}$is qualitatively 

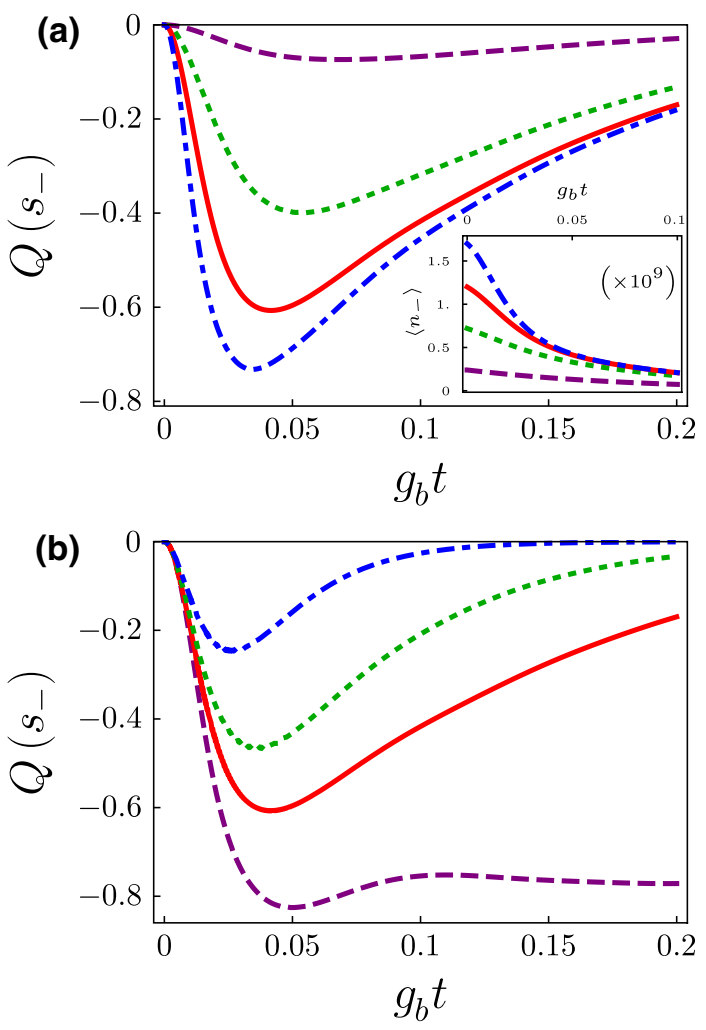

FIG. 9. With the realistic parameters $U=8.5 \times 10^{-8}, g_{c}=$ $60 \mathrm{~m}^{-1}$, tail length $N=28$, and the optimal coupling ratio Eq. (12), mode $s_{-}$quickly evolves to a strongly sub-Poissonian state. A coherent state with average photon number $n_{-}(0)$ is initialized in mode $s_{-}$, and all other modes are initialized into the vacuum. (a) The dot-dashed, solid, dotted, and dashed lines correspond to $\left\langle n_{-}(0)\right\rangle=1.7 \times 10^{9}, 1.2 \times 10^{9}, 7.2 \times 10^{8}$, and $2.4 \times$ $10^{8}$, respectively, and $\gamma_{1}=11.5 \mathrm{~m}^{-1}$. Inset, the evolution of photon number $\left\langle n_{-}\right\rangle$displays the nonlinear decay behavior perculiar to the NCL mechanism. (b) The Mandel parameter $Q$ remains strongly negative even in the presence of realistic linear loss $\gamma_{1}$. The dashed, solid, dotted, and dot-dashed lines correspond to $\gamma_{1}=0,11.5,20$, and $40 \mathrm{~m}^{-1}$, respectively, and $\left\langle n_{-}(0)\right\rangle=$ $1.2 \times 10^{9}$.

equivalent to Fig. 9, but a slightly different optimal ratio $g_{b} / g_{a}$ should be considered.

Finally, we note that in the case of $\gamma_{1}=0$, the signal modes are highly sensitive to whether $N$ is odd or even, and for even $N$ the decay of mode $s_{-}$is inhibited. Indeed, the decay rate $\gamma^{\prime}$ into an effective reservoir changes depending on $N$, and may be understood as an interference effect resulting from the unitary coupling between tail modes affecting whether the excitation returns to signal mode $a$ or mode $b$ from the tail. This effect of the reservoir, which quickly vanishes at even small levels of linear loss $\gamma_{1}$, will not manifest itself over the timescales of interest provided that $N$ is large.

We demonstrate that the multimode model—which is the model most closely aligned to a physical

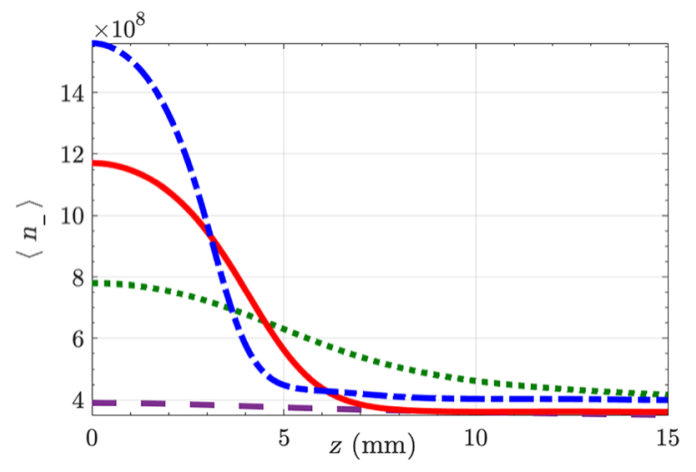

FIG. 10. Evolution of photon number from the numerical solution of the 100-fs pulse propagation in the waveguide structure with five modes in the tail. We account explicitly for chromatic dispersion, Kerr effect and self-steepening, and solve the nonlinear Schrödinger equation via the split-step Fourier method for the system of coupled waveguides. The blue, red, green, and purple lines correspond to $\left\langle n_{-}(0)\right\rangle=1.6 \times 10^{9}, 1.2 \times 10^{9}, 7.8 \times 10^{8}$, and $3.9 \times 10^{8}$. The evolution of the photon-number characteristic for the NCL mechanism is observed [compare with Fig. 9(a)].

implementation via waveguides inscribed in bulk glass, Fig. 2(a) - can generate a strongly sub-Poissonian output from a classical input state into mode $s_{-}$over the short timescales of initial evolution. This corroborates results from Sec. III and the prediction of optimal coupling parameters $g_{a, b}$ Eq. (12) when mode $s_{-}$is initially excited.

To assess the impact of specific effects of short-pulse propagation in the system of coupled waveguides, we dynamically evolved the spectral and temporal properties of the pulse and analyzed their influence on the effectiveness of the NCL mechanism. In particular, we consider the combined effect of chromatic dispersion, self-phase modulation (Kerr effect), and self-steepening of the pulse via the nonlinear Schrödinger equation (NLSE) for the system of waveguides in a $\chi^{(3)}$ nonlinear medium. The resulting system of coupled propagation equations for all waveguides in the PhoG structure is numerically solved using the split-step Fourier method [46]. For a specific comparison we use the signature of the NCL mechanism, the nonlinear decay behavior of photon number $\left\langle n_{-}\right\rangle$in the antisymmetric mode $s_{-}$displayed in the inset of Fig. 9(a), which may additionally be interpreted as an intensity-dependent loss. In Fig. 10 such a decay can be clearly seen when evolving an initially Gaussian pulse according to the NLSE. Linear loss is neglected in this simulation to ensure that the decay is caused by the NCL mechanism, though we note that even when linear loss is included we observe strong agreement between the nonlinear decay behavior of the full pulse propagation (NLSE) numerics and the quantum multimode model considered in Fig. 9.

The pulse propagation is modeled for a $100-$ fs initially Gaussian pulse in IG2 glass with a central wavelength of $1550 \mathrm{~nm}$ (see Ref. [34], and also Sec. III C 1). Initial 
pulse energies of 50,100, 150, and $200 \mathrm{pJ}$ are chosen, in order to compare with the inset of Fig. 9(a). The nonlinear parameter $\bar{\gamma}^{\mathrm{NL}}$ is set at $0.6 \mathrm{~W}^{-1} \mathrm{~m}^{-1}$, considering that the pulse energy is $200 \mathrm{pJ}$. The resulting evolution is displayed in Fig. 10 and we observe the characteristic nonlinear decay, which is a good evidence that NCL occurs for these realistic physical parameters even when the full pulse dynamics is considered. Linear loss is neglected in this simulation to ensure that the decay is caused by NCL mechanism.

It is found that $g_{a}=210 \mathrm{~m}^{-1}, g_{b}=360 \mathrm{~m}^{-1}$, and $g_{c}=$ $430 \mathrm{~m}^{-1}$ best reproduces the signature behavior of NCL (different decaying rates for the different initial energies) as shown in Fig. 10. The optimal ratio between interaction constants is only weakly influenced by the effects of the pulse propagation. The spectral broadening of the pulse is estimated to be about $10 \mathrm{~nm}$ for $100-\mathrm{fs}$ input pulse and $2-3-\mathrm{cm}$ device at $1550-\mathrm{nm}$ central wavelength. Provided that the device length is sufficient for the shortest wavelength of the pulse to undergo NCL decay (and for the example shown in Fig. 10 it is sufficient), the effects of such pulse spectral broadening do not affect the manifestations of the NCL mechanism.

\section{CONCLUSIONS}

We discuss the design of a family of PhoG-based devices of coupled single-mode waveguides in bulk nonlinear glass. We show that an asymmetric structure can indeed function as a deterministic generator of bright sub-Poissonian states of light. The sub-Poissonian light generation with considerably large photon-number squeezing (up to the values of the Mandel parameter of about -0.8 ) can occur at the initial stages of the dynamics. In this regime conventional linear loss (which is quite high in nonlinear glass) still can be overcome by sufficiently intensive coherent input, and the nonclassical states can be produced. A hierarchy of models has been derived, from the original multimode waveguide network down to the simplest single-mode model exhibiting both two-photon and nonlinear-coherent loss. We develop an analytic approach to the single-mode model and discovered two dimensionless parameters allowing one to design the waveguide system with the aim of reaching a maximally nonclassical state for a given length of the structure. Feasibility of the suggested device has been analyzed for three different eligible practical structures: a system of waveguides in bulk glass, multicore fiber, a set of coupled nanowires. We estimate the number of photons required to reach large nonclassicality with the femtosecond input pulses and demonstrate that this number remains well within the operational possibilities. Further, we develop an approximation linearized on the quantum correction to the classical solution and demonstrate its closeness with the exact solution of the single-mode problem. Both the three-mode approximation and the complete multimode model lead to very close results in the initial stage of the dynamics.

In summary, suggested CDP circuits indeed allow for implementation of the engineered nonlinear loss to produce strongly nonclassical states in realistic photonic structures. Our design of the PhoG with a system of waveguides laser written in the bulk glass can serve as a practical recipe for realizing such structures. Being comparatively inexpensive and easy to produce, these generators can find applications for different detection, measurement, and metrology tasks [22,23] and as quasi-single-photon sources in various quantum-technology applications, such as, e.g., quantum-key distribution [21].

\section{ACKNOWLEDGMENTS}

The authors acknowledge support from the EU Flagship on Quantum Technologies, project PhoG (820365). D.M., A.S., and A.M. also acknowledge support from the National Academy of Sciences of Belarus program "Convergence," and the BRRFI project F18U-006. We thank Benjamin Brecht for the help in developing the code for pulse propagation in waveguide network.

\section{APPENDIX A: ON GAUSSIANITY OF THE PHOTON-NUMBER DISTRIBUTION OF THE PHOG GENERATED STATE}

Here we derive approximate analytical expressions for dynamics of mean photon number, Mandel parameter, and higher-order moments of the photon-number distribution for the single-mode model. We start from the master equation (8) of the main text. For the photon-number distribution, $p(n)=\langle n|\rho| n\rangle$, Eq. (8) yields

$$
\frac{d p(n)}{d z}=\sum_{i=1}^{3} \gamma_{i}\left[f_{i}\left(n+\xi_{i}\right) p\left(n+\xi_{i}\right)-f_{i}(n) p(n)\right],
$$

where $\xi_{i}$ is the number of photons lost per each dissipation event $\left(\xi_{1}=\xi_{3}=1, \xi_{2}=2\right) ; f_{1}(n)=n, f_{2}(n)=n(n-1)$, and $f_{3}(n)=n(n-1)^{2}$.

For a function $g(n)$, one can define its average as

$$
\langle g(n)\rangle=\sum_{n=0}^{\infty} g(n) p(n) .
$$

Equation (A1) implies that the average satisfies the following equation:

$$
\frac{d}{d z}\langle g(n)\rangle=\sum_{i=1}^{3} \gamma_{i}\left\langle f_{i}(n)\left[g\left(n-\xi_{i}\right)-g(n)\right]\right\rangle .
$$


For example, the mean photon number $\mu=\langle n\rangle$ corresponds to $g(n)=n$ and satisfies

$$
\begin{aligned}
\frac{d \mu}{d z} & =-\sum_{i=1}^{3} \gamma_{i} \xi_{i}\left\langle f_{i}(n)\right\rangle \\
& =-\gamma_{1} \mu-2 \gamma_{2}\left(\left\langle n^{2}\right\rangle-\mu\right)-\gamma_{3}\left(\left\langle n^{3}\right\rangle-2\left\langle n^{2}\right\rangle+\mu\right) .
\end{aligned}
$$

For further calculation of the photon-number distribution moments, it is also useful to calculate the derivative for a function $g(\delta n)$ of the photon-number deviation $\delta n=n-$ $\mu$. Here, one needs to take into account that $\mu$ also varies with $z$ :

$$
\begin{aligned}
\frac{d}{d z}\langle g(\delta n)\rangle= & \sum_{i=1}^{3} \gamma_{i}\left\langle f_{i}(n)\left[g\left(\delta n-\xi_{i}\right)-g(\delta n)\right]\right\rangle \\
& -\left\langle g^{\prime}(\delta n)\right\rangle \frac{d \mu}{d z}, \\
= & \sum_{i=1}^{3} \gamma_{i}\left\{\left\langle f_{i}(n)\left[g\left(\delta n-\xi_{i}\right)-g(\delta n)\right]\right\rangle\right. \\
& \left.+\xi_{i}\left\langle g^{\prime}(\delta n)\right\rangle\left\langle f_{i}(n)\right\rangle\right\} .
\end{aligned}
$$

In further calculations, $n$ in Eq. (A5) can also be expressed in terms of $\delta n: n=\mu+\delta n$.

Let us assume that the initial state is a coherent one with the mean photon number $\mu(z=0)=n_{0}$ :

$$
p(n, z=0)=\frac{n_{0}^{n}}{n !} e^{-n_{0}}
$$

Central moments of the input state are equal to

$$
\left\langle\delta n^{2}\right\rangle=\mu, \quad\left\langle\delta n^{3}\right\rangle=\mu, \quad\left\langle\delta n^{4}\right\rangle=3 \mu^{2}+\mu .
$$

Taking into account that Poisson distribution does not have heavy tails and its central moments are mainly determined by the central part of the distribution, one may expect that, at least for the beginning of the state evolution, $p(n)$ will have nonzero values for $|\delta n| \lesssim$ const $\sqrt{\mu}$, and, therefore,

$$
\left\langle\delta n^{k}\right\rangle=O\left(\mu^{k / 2}\right)
$$

Let us consider such $z$ that conditions (A8), together with

$$
\zeta_{2}=\frac{\left\langle\delta n^{2}\right\rangle}{\mu}=O(1)
$$

are still satisfied. Substituting the definition of $\zeta_{2}$ into Eq. (A5) and using Eq. (A8) for $k \geq 3$, one can derive the following equation:

$$
\begin{aligned}
\frac{d \zeta_{2}}{d z}= & \gamma_{1}\left(1-\zeta_{2}\right) \\
& +2 \gamma_{2}\left\{\left(2-3 \zeta_{2}\right) \mu+O(\sqrt{\mu})\right\} \\
& +\gamma_{3}\left\{\left(1-5 \zeta_{2}\right) \mu^{2}+O\left(\mu^{3 / 2}\right)\right\}
\end{aligned}
$$

According to the derived equation, the quantity $\zeta_{2}$ tends to reach the value

$$
\zeta_{2}^{(f)} \approx \frac{\gamma_{1}+4 \gamma_{2} \mu+\gamma_{3} \mu^{2}}{\gamma_{1}+6 \gamma_{2} \mu+5 \gamma_{3} \mu^{2}}=O(1)
$$

Taking into account that $\zeta_{2}(z=0)=1$, one can expect that condition (A8) will be satisfied during most of the system evolution, until the mean photon number $\mu$ becomes small.

Assuming that a single type of dissipation is prevailing, one can derive the values

$$
\zeta_{2}^{(f)}=1,2 / 3,1 / 5
$$

for linear, two-photon, and nonlinear coherent loss, respectively. The corresponding values of Mandel parameter $Q=$ $\zeta_{2}-1$ are

$$
Q=0,-1 / 3,-4 / 5
$$

Now let us assume that for the considered $z$ the conditions

$$
\zeta_{3}=\frac{\left\langle\delta n^{3}\right\rangle}{\mu}=O(1)
$$

and

$$
\zeta_{4}=\frac{\left\langle\delta n^{4}\right\rangle-3 \zeta_{2} \mu}{\mu^{3 / 2}}=O(1)
$$

are still satisfied (for $z=0$ one has $\zeta_{3}=1$ and $\zeta_{4}=$ $1 / \sqrt{\mu}$ ) together with Eq. (A8) for $k \geq 5$.

Therefore, one can derive the following equation for $\zeta_{3}$ :

$$
\begin{aligned}
\frac{d \zeta_{3}}{d z}= & \gamma_{1}\left(-1+3 \zeta_{2}-2 \zeta_{3}\right) \\
& +2 \gamma_{2}\left\{\left(-4+12 \zeta_{2}-6 \zeta_{2}^{2}-5 \zeta_{3}\right) \mu+O(\sqrt{\mu})\right\} \\
& +\gamma_{3}\left\{\left(-1+9 \zeta_{2}-18 \zeta_{2}^{2}-8 \zeta_{3}\right) \mu^{2}+O\left(\mu^{3 / 2}\right)\right\}
\end{aligned}
$$

The quantity $\zeta_{3}$ tends to the value

$$
\begin{aligned}
\zeta_{3}^{(f)} \approx & \frac{1}{2 \gamma_{1}+10 \gamma_{2} \mu+8 \gamma_{3} \mu^{2}}\left[\gamma_{1}\left(3 \zeta_{2}-1\right)\right. \\
& +4 \gamma_{2} \mu\left(-2+6 \zeta_{2}-3 \zeta_{2}^{2}\right) \\
& \left.+\gamma_{3} \mu^{2}\left(-1+9 \zeta_{2}-18 \zeta_{2}^{2}\right)\right]=O(1)
\end{aligned}
$$


In a similar way one can show that $\zeta_{4}$ satisfies

$$
\begin{aligned}
\frac{d \zeta_{4}}{d z}= & \gamma_{1}\left\{-4 \zeta_{4} \sqrt{\mu}+O(1)\right\} \\
& +2 \gamma_{2}\left\{-8\left(2 \zeta_{4}+\frac{\left\langle\delta n^{5}\right\rangle}{\mu^{5 / 2}}\right) \mu^{3 / 2}+O(\mu)\right\} \\
& +\gamma_{3}\left\{-12\left(\zeta_{4}++\frac{\left\langle\delta n^{5}\right\rangle}{\mu^{5 / 2}}\right) \mu^{5 / 2}+O\left(\mu^{2}\right)\right\} .
\end{aligned}
$$

The derived equation has two important implications. First, it shows that the condition $\zeta_{4}=O(1)$ remains valid while $\left\langle\delta n^{5}\right\rangle / \mu^{5 / 2}=O(1)$, which in its turn is a consequence of Eqs. (A10) and (A11). Second, in contrast to lowerorder moments, the equation for $\zeta_{4}$ is not disentangled from higher moments and cannot be solved independently of them. Still, the condition $\zeta_{4}=O(1)$ is sufficient for validity of the previously derived Eqs. (A16) and (A11).

For a single type of dissipation, one can derive the values

$$
\zeta_{3}^{(f)}=1,2 / 30,1 / 100
$$

for linear, two-photon, and nonlinear coherent loss respectively, which correspond to the following values of skew$\operatorname{ness} \zeta_{3} /\left(\sqrt{\mu} \zeta_{2}^{3 / 2}\right)$ :

$$
\text { Skewness }=\frac{1}{\sqrt{\mu}}, \frac{0.12}{\sqrt{\mu}}, \frac{0.11}{\sqrt{\mu}} .
$$

Similarly, for excess kurtosis one can derive the estimate

$$
\text { Excess kurtosis }=\frac{\left\langle\delta n^{4}\right\rangle}{\mu^{2} \zeta_{2}^{2}}-3=\frac{\zeta_{4}}{\sqrt{\mu} \zeta_{2}^{2}}=O\left(\frac{1}{\sqrt{\mu}}\right) .
$$

The results show that the photon-number distribution, despite becoming strongly sub-Poissonian, remain Gaussian with high accuracy until the mean photon number $\mu$ becomes small.

\section{APPENDIX B: DERIVATION OF LINEARIZATION APPROXIMATIONS}

In this section we derive the linearization approximations, which are used in Secs. III D and VI. These approximations become increasingly necessary for modeling the system when large photon numbers or large numbers of modes must be considered.

Consider arbitrary quantum operators $A, B, C$. We explicitly show the derivation for linearization of $\langle A B C\rangle$, but the approach may be readily generalized to higherorder products. We derive replacements, which allow us to approximate expectations of products of three or more operators, using only first- and second-order terms, e.g., $\langle A\rangle,\langle A B\rangle$.

We may expand each operator $A, B, C$ into a classical "mean-field" term and a quantum fluctuation term: $A=\langle A\rangle+\delta A$. We take $\langle\delta A\rangle=0$, which ensures that the mean-field term $\langle A\rangle$ is meaningful and consistent. Then substituting these expansions into $\langle A B C\rangle$,

$$
\begin{aligned}
\langle A B C\rangle= & \langle A\rangle\langle B\rangle\langle C\rangle+\langle A\rangle\langle\delta B \delta C\rangle+\langle B\rangle\langle\delta A \delta C\rangle \\
& +\langle C\rangle\langle\delta A \delta B\rangle+\langle\delta A \delta B \delta C\rangle .
\end{aligned}
$$

A key tool we require is the cumulant expansion for generic operators $\left\{O_{1}, \ldots, O_{n}\right\}$

$$
\mathcal{C}\left(O_{1}, \ldots, O_{n}\right)=\sum_{\mathcal{P} \in \mathbb{P}}(|\mathcal{P}|-1) !(-1)^{|\mathcal{P}|-1} \prod_{p \in \mathcal{P}}\left\langle\prod_{i \in p} O_{i}\right\rangle,
$$

where $\mathbb{P}$ denotes all disjoint partitions of the set of operators, $|\mathcal{P}|$ denotes the number of blocks in partition $\mathcal{P}$, and $p$ iterates over each block in the partition. For example,

$$
\begin{aligned}
\mathcal{C}(X, Y, Z)= & \langle X Y Z\rangle+2\langle X\rangle\langle Y\rangle\langle Z\rangle-\langle X\rangle\langle Y Z\rangle \\
& -\langle Y\rangle\langle X Z\rangle-\langle Z\rangle\langle X Y\rangle .
\end{aligned}
$$

We perform our linearization assumption on Eq. (B1) by setting $\mathcal{C}(\delta A, \delta B, \delta C)=0$, which implies therefore that $\langle\delta A \delta B \delta C\rangle=0$ since $\langle\delta A\rangle=\langle\delta B\rangle=\langle\delta C\rangle=0$. Finally, using $\delta A=A-\langle A\rangle$ we arrive at our final expression,

$$
\begin{aligned}
\langle A B C\rangle \approx & \langle A\rangle\langle B C\rangle+\langle B\rangle\langle A C\rangle+\langle C\rangle\langle A B\rangle \\
& -2\langle A\rangle\langle B\rangle\langle C\rangle .
\end{aligned}
$$

Higher-order expectations of products of operators may be calculated in the same way, with the only requirements assumed about fluctuations being the zero-mean condition $\langle\delta A\rangle=\ldots=\langle\delta Z\rangle=0$ and the linearization approximation $\mathcal{C}(\delta A, \ldots, \delta Z)=0$.

\section{APPENDIX C: LINEARIZED SINGLE-MODE MODEL}

By applying the linearization approximations derived in Appendix B to the system of coupled ODEs (21) we arrive at the following closed system of ODEs:

$$
\begin{aligned}
\partial_{t}\left\langle s_{-}\right\rangle= & c_{1}\left\langle s_{-}\right\rangle+c_{2}\left(\left\langle s_{-}^{\dagger}\right\rangle\left\langle s_{-}^{2}\right\rangle+2\left\langle s_{-}\right\rangle\left\langle n_{-}\right\rangle-2\left\langle s_{-}^{\dagger}\right\rangle\left\langle s_{-}\right\rangle^{2}\right) \\
& -\frac{\gamma_{3}}{2}\left(6\left\langle s_{-}^{\dagger}\right\rangle\left\langle n_{-}\right\rangle\left\langle s_{-}^{2}\right\rangle+3\left\langle s_{-}\right\rangle\left\langle s_{-}^{\dagger}\right\rangle\left\langle s_{-}^{2}\right\rangle\right. \\
& +6\left\langle s_{-}\right\rangle\left\langle n_{-}\right\rangle^{2}-2\left\langle s_{-}^{\dagger 2}\right\rangle\left\langle s_{-}\right\rangle^{3}-12\left\langle n_{-}\right\rangle\left\langle s_{-}^{\dagger}\right\rangle\left\langle s_{-}\right\rangle^{2} \\
& \left.-6\left\langle s_{-}^{2}\right\rangle\left\langle s_{-}^{\dagger}\right\rangle^{2}\left\langle s_{-}\right\rangle+6\left\langle s_{-}^{\dagger}\right\rangle^{2}\left\langle s_{-}\right\rangle^{3}\right)
\end{aligned}
$$




$$
\begin{aligned}
\partial_{t}\left\langle s_{-}^{\dagger}\right\rangle= & c_{1}^{*}\left\langle s_{-}^{\dagger}\right\rangle+c_{2}^{*}\left(\left\langle s_{-}\right\rangle\left\langle s_{-}^{\dagger 2}\right\rangle+2\left\langle s_{-}^{\dagger}\right\rangle\left\langle n_{-}\right\rangle-2\left\langle s_{-}^{\dagger}\right\rangle^{2}\left\langle s_{-}\right\rangle\right) \\
& -\frac{\gamma_{3}}{2}\left(6\left\langle s_{-}\right\rangle\left\langle n_{-}\right\rangle\left\langle s_{-}^{\dagger 2}\right\rangle+3\left\langle s_{-}^{\dagger}\right\rangle\left\langle s_{-}^{2}\right\rangle\left\langle s_{-}^{\dagger 2}\right\rangle\right. \\
& +6\left\langle s_{-}^{\dagger}\right\rangle\left\langle n_{-}\right\rangle^{2}-2\left\langle s_{-}^{2}\right\rangle\left\langle n_{-}^{3}\right\rangle-12\left\langle n_{-}\right\rangle\left\langle s_{-}\right\rangle\left\langle s_{-}\right\rangle^{2} \\
& \left.-6\left\langle s_{-}^{\dagger 2}\right\rangle\left\langle s_{-}\right\rangle^{2}\left\langle s_{-}^{\dagger}\right\rangle+6\left\langle s_{-}^{\dagger 3}\right\rangle\left\langle s_{-}\right\rangle^{2}\right) \\
\partial_{t}\left\langle s_{-}^{2}\right\rangle= & c_{3}\left\langle s_{-} s_{-}\right\rangle+c_{4}\left(3\left\langle n_{-}\right\rangle\left\langle s_{-}^{2}\right\rangle-2\left\langle s_{-}^{\dagger}\right\rangle\left\langle s_{-}\right\rangle^{3}\right) \\
& -\gamma_{3}\left(3\left\langle s_{-}^{\dagger 2}\right\rangle\left\langle s_{-}^{2}\right\rangle^{2}+12\left\langle n_{-}\right\rangle^{2}\left\langle s_{-}^{2}\right\rangle-2\left\langle s_{-}^{\dagger 2}\right\rangle\left\langle s_{-}\right\rangle^{4}\right. \\
& -12\left\langle s_{-}^{2}\right\rangle\left\langle s_{-}^{\dagger}\right\rangle^{2}\left\langle s_{-}\right\rangle^{2}-16\left\langle n_{-}\right\rangle\left\langle s_{-}^{\dagger}\right\rangle\left\langle s_{-}\right\rangle^{3} \\
& \left.+16\left\langle s_{-}^{\dagger}\right\rangle^{2}\left\langle s_{-}\right\rangle^{4}\right) \\
\partial_{t}\left\langle s_{-}^{\dagger^{2}}\right\rangle= & c_{3}^{*}\left\langle s_{-}^{\dagger 2}\right\rangle+c_{4}^{*}\left(3\left\langle s_{-}^{\dagger 2}\right\rangle\left\langle n_{-}\right\rangle-2\left\langle s_{-}^{\dagger}\right\rangle^{3}\left\langle s_{-}\right\rangle\right) \\
& -\gamma_{3}\left(3\left\langle s_{-}^{\dagger 2}\right\rangle^{2}\left\langle s_{-}^{2}\right\rangle+12\left\langle s_{-}^{\dagger 2}\right\rangle\left\langle n_{-}\right\rangle^{2}-2\left\langle s_{-}^{2}\right\rangle\left\langle s_{-}^{\dagger}\right\rangle^{4}\right. \\
& -21\left\langle s_{-}^{\dagger 2}\right\rangle\left\langle s_{-}^{\dagger}\right\rangle^{2}\left\langle s_{-}\right\rangle^{2}-16\left\langle n_{-}\right\rangle\left\langle s_{-}^{\dagger}\right\rangle^{3}\left\langle s_{-}\right\rangle \\
& \left.+16\left\langle s_{-}^{\dagger}\right\rangle^{4}\left\langle s_{-}\right\rangle^{2}\right) \\
\partial_{t}\left\langle n_{-}\right\rangle= & -\gamma_{1}\left\langle n_{-}\right\rangle+c_{5}\left(\left\langle s_{-}^{\dagger 2}\right\rangle\left\langle s_{-}^{2}\right\rangle+2\left\langle n_{-}\right\rangle^{2}-2\left\langle s_{-}^{\dagger}\right\rangle^{2}\left\langle s_{-}\right\rangle^{2}\right) \\
& -\gamma_{3}\left(9\left\langle s_{-}^{\dagger 2}\right\rangle\left\langle n n_{-}\right\rangle\left\langle s_{-}^{2}\right\rangle+6\left\langle n_{-}\right\rangle^{3}-6\left\langle s_{-}^{\dagger 2}\right\rangle\left\langle s_{-}^{\dagger}\right\rangle\left\langle s_{-}\right\rangle^{3}\right. \\
& -18\left\langle n_{-}\right\rangle\left\langle s_{-}^{\dagger}\right\rangle^{2}\left\langle s_{-}\right\rangle^{2}-6\left\langle s_{-}^{2}\right\rangle\left\langle s_{-}^{\dagger}\right\rangle^{3}\left\langle s_{-}\right\rangle \\
& \left.+16\left\langle s_{-}^{\dagger}\right\rangle^{3}\left\langle s_{-}\right\rangle^{3}\right), \\
& (\mathrm{C} 5)
\end{aligned}
$$

with $n_{-}=s_{-}^{\dagger} s_{-}$.

This system is solved numerically for $\left\langle s_{-}\right\rangle,\left\langle s_{-}^{\dagger}\right\rangle,\left\langle s_{-}^{2}\right\rangle$, $\left\langle s_{-}^{\dagger 2}\right\rangle,\left\langle n_{-}\right\rangle$and the results are shown as dashed lines in Fig. 5.

[1] M. Wolinsky and H. J. Carmichael, Quantum Noise in the Parametric Oscillator: From Squeezed States to Coherent-State Superpositions, Phys. Rev. Lett. 60, 1836 (1988).

[2] F. Verstraete, M. M. Wolf, and I. J. Cirac, Quantum computation, quantum-state engineering, and quantum phase transitions driven by dissipation, Nat. Phys. 5, 633 (2009).

[3] A. Metelmann and A. A. Clerk, Nonreciprocal Photon Transmission and Amplification via Reservoir Engineering, Phys. Rev. X 5, 021025 (2015).

[4] D. N. Biggerstaff et al., Enhancing coherent transport in a photonic network using controllable decoherence, Nat. Commun. 7, 11282 (2016).

[5] S. Clark, A. Peng, M. Gu, and S. Parkins, Unconditional Preparation of Entanglement between Atoms in Cascaded Optical Cavities, Phys. Rev. Lett. 91, 177901 (2003).
[6] J. F. Poyatos, J. I. Cirac, and P. Zoller, Quantum Reservoir Engineering with Laser Cooled Trapped Ions, Phys. Rev. Lett. 77, 4728 (1996).

[7] J. T. Barreiro, M. Muller, Ph. Schindler, D. Nigg, Th. Monz, M. Chwalla, M. Hennrich, Ch. F. Roos, P. Zoller, and R. Blat, An open-system quantum simulator with trapped ions, Nature 470, 486 (2011).

[8] A. W. Carr and M. Saffman, Preparation of Entangled and Antiferromagnetic States by Dissipative Rydberg Pumping, Phys. Rev. Lett. 111, 033607 (2013).

[9] M. Roghani and H. Weimer, Dissipative preparation of entangled many-body states with Rydberg atoms, Quantum Sci. Technol. 3, 035002 (2018).

[10] Z. Leghtas, S. Touzard, I. M. Pop, A. Kou, B. Vlastakis, A. Petrenko, K. M. Sliwa, A. Narla, S. Shankar, M. J. Hatridge, M. Reagor, L. Frunzio, R. J. Schoelkopf, M. Mirrahimi, and M. H. Devoret, Confining the state of light to a quantum manifold by engineered two-photon loss, Science 347, 853 (2015).

[11] M. E. Kimchi-Schwartz, L. Martin, E. Flurin, C. Aron, M. Kulkarni, H. E. Tureci, and I. Siddiqi, Stabilizing Entanglement via Symmetry-Selective Bath Engineering in Superconducting Qubits, Phys. Rev. Lett. 116, 240503 (2016).

[12] E. Kapit, The upside of noise: Engineered dissipation as a resource in superconducting circuits, Quantum Sci. Technol. 2, 033002 (2017).

[13] S. Mukherjee, A. Spracklen, D. Choudhury, N. Goldman, P. Öhberg, E. Andersson, and R. R. Thomson, Observation of a Localized Flat-Band State in a Photonic Lieb Lattice, Phys. Rev. Lett. 114, 245504 (2015).

[14] R. A. Vicencio, C. Cantillano, L. Morales-Inostroza, B. Real, C. Mejía-Cortés, S. Weimann, A. Szameit, and M. I. Molina, Observation of Localized States in Lieb Photonic Lattices, Phys. Rev. Lett. 114, 245503 (2015).

[15] S. Mukherjee, D. Mogilevtsev, G. Y. Slepyan, T. H. Doherty, R. R. Thomson, and N. Korolkova, Dissipatively coupled waveguide networks for coherent diffusive photonics, Nat. Comm. 8, 1909 (2017).

[16] M. D. Eisamana, J. Fan, A. Migdall, and S. V. Polyakov, Invited review article: Single-photon sources and detectors, Rev. Sci. Instrum. 82, 071101 (2011).

[17] P. Senellart, G. Solomon, and A. White, High-performance semiconductor quantum-dot single-photon sources, Nat. Nanotechnol. 12, 1026 (2017).

[18] A. Lohrmann, B. C. Johnson, J. C. McCallum, and S. Castelletto, A review on single photon sources in silicon carbide, Rep. Prog. Phys. 80, 034502 (2017).

[19] N. Somaschiet al. , Near-optimal single-photon sources in the solid state, Nat. Photonics 10, 340 (2016); L. Dusanowski, S.-H. Kwon, Ch. Schneider, and S. Höfling, Near-Unity Indistinguishability Single Photon Source for Large-Scale Integrated Quantum Optics, Phys. Rev. Lett. 122, 173602 (2019).

[20] X. B. Wang, C. Z. Peng, J. Zhang, L. Yang, and J. W. Pan, General theory of decoy-state quantum cryptography with source errors, Phys. Rev. A 77, 042311 (2008).

[21] A. Li, T. Chen, Y. Zhou, and X. Wang, On-demand singlephoton sources via quantum blockade and applications in decoy-state quantum key distribution, Opt. Lett. 41, 1921 (2016). 
[22] M. A. Taylor, J. Janousek, V. Daria, J. Knittel, B. Hage, H.-A. Bachor, and W. P. Bowen, Biological measurement beyond the quantum limit, Nat. Phot. 7, 229 (2013).

[23] I. Ruo Berchera and I. P. Degiovanni, Quantum imaging with sub-poissonian light: Challenges and perspectives in optical metrology, Metrologia 56, 024001 (2019).

[24] D. Mogilevtsev and V. S. Shchesnovich, Single-photon generation by correlated loss in a three-core optical fiber, Opt. Lett. 35, 3375 (2010).

[25] V. S. Shchesnovich and D. Mogilevtsev, Generators of nonclassical states by a combination of linear coupling of boson modes, Kerr nonlinearity, and strong linear losses, Phys. Rev. A 84, 013805 (2011).

[26] V. I. Manko, G. Marmo, E. C. G. Sudarshan, and F. Zaccaria, f-Oscillators and nonlinear coherent states, Phys. Scr. 55, 528 (1997).

[27] R. L. de Matos Filho and W. Vogel, Nonlinear coherent states, Phys. Rev. A 54, 4560 (1996).

[28] H. Ezaki, E. Hanamura, and Y. Yamamoto, Generation of Phase States by Two-Photon Absorption, Phys. Rev. Lett. 83, 3558 (1999).

[29] M. Alexanian and S. K. Bose, Comment on "Generation of Phase States by Two-Photon Absorption”, Phys. Rev. Lett. 85, 1136 (2000).

[30] L. Davidovich, Sub-poissonian processes in quantum optics, Rev. Mod. Phys. 68, 127 (1996).

[31] D. Mogilevtsev, A. Mikhalychev, V. S. Shchesnovich, and Natalia Korolkova, Nonlinear dissipation can combat linear loss, Phys. Rev. A 87, 063847 (2013).

[32] P. D. Drummond and D. F. Walls, Quantum theory of optical bistability. I. Nonlinear polarisability model, J. Phys. A: Math. Gen. 13, 725 (1980).

[33] R. del Coso and J. Solis, Relation between nonlinear refractive index and third-order susceptibility in absorbing media, J. Opt. Soc. Am. B 21, 640 (2004).

[34] Optical constants of Vitron IG2, https://refractiveindex. info/?shelf=glass\&book=VITRON-IG\&page=IG2.
[35] H. L. Butcher, D. G. MacLachlan, D. Lee, R. R. Thomson, and D. Weidmann, Demonstration and characterization of ultrafast laser-inscribed mid-infrared waveguides in chalcogenide glass IG2,

[36] A. Hosaka, K. Hirosawa, R. Sawada, and F. Kannari, Generation of photon-number squeezed states with a fiber-optic symmetric interferometer, Opt. Exp. 15, 18850 (2015).

[37] T. Sato, S. Makino, Y. Ishizaka, T. Fujisawa, and K. Saitoh, A rigorous definition of nonlinear parameter $\gamma$ and effective area $A_{\text {eff }}$ for photonic crystal optical waveguides, JOSA B 32, 1245 (2015). Opt. Exp. 26, 10930 (2018).

[38] M. Alexanian and S. K. Bose, Comment on "Generation of Phase States by Two-Photon Absorption," Phys. Rev. Lett. 85, 1136 (2000); H. Ezaki, E. Hanamura, and Y. Yamamoto, Ezaki et al. Reply, Phys. Rev. Lett. 85, 1137 (2000).

[39] C. Weedbrook, S. Pirandola, R. García-Patrón, N. J. Cerf, T. C. Ralph, J. H. Shapiro, and S. Lloyd, Gaussian quantum information, Rev. Mod. Phys. 84, 621 (2012).

[40] G. Adesso, Entanglement of Gaussian states, arXiv:quant$\mathrm{ph} / 0702069 \mathrm{v} 1$.

[41] R. Simon, Peres-Horodecki Separability Criterion for Continuous Variable Systems, Phys. Rev. Lett. 84, 2726 (2000).

[42] J. E. Sharping, K. F. Lee, M. A. Foster, A. C. Turner, B. S. Schmidt, M. Lipson, A. L. Gaeta, and P. Kumar, Generation of correlated photons in nanoscale silicon waveguides, Opt. Express 14, 12388 (2006).

[43] H. Takesue, Y. Tokura, H. Fukuda, T. Tsuchizawa, T. Watanabe, K. Yamada, and S.-I. Itabashi, Entanglement generation using silicon wire waveguide, Appl. Phys. Lett. 91, 201108 (2007).

[44] Devices Based on Low-Dimensional Semiconductor Structures, edited by M. Balkanski, Nato Science Partnership Subseries: 3 (Springer, Netherlands, 1996), Vol. 14.

[45] M. B. Plenio and P. L. Knight, The quantum-jump approach to dissipative dynamics in quantum optics, Rev. Mod. Phys. 70, 101 (1998).

[46] G. Agrawal, Nonlinear Fiber Optics (Academic Press, San Diego, 2001), 3rd ed. 